\title{
Balance Training Programs in Athletes - A Systematic Review
}

\author{
by \\ Anna Brachman ${ }^{1}$, Anna Kamieniarz'1, Justyna Michalska1, Michał Pawłowski \\ Kajetan J. Słomka1, Grzegorz Juras ${ }^{1}$
}

It has become almost routine practice to incorporate balance exercises into training programs for athletes from different sports. However, the type of training that is most efficient remains unclear, as well as the frequency, intensity and duration of the exercise that would be most beneficial have not yet been determined. The following review is based on papers that were found through computerized searches of PubMed and SportDiscus from 2000 to 2016. Articles related to balance training, testing, and injury prevention in young healthy athletes were considered. Based on a Boolean search strategy the independent researchers performed a literature review. A total of 2395 articles were evaluated, yet only 50 studies met the inclusion criteria. In most of the reviewed articles, balance training has proven to be an effective tool for the improvement of postural control. It is difficult to establish one model of training that would be appropriate for each sport discipline, including its characteristics and demands. The main aim of this review was to identify a training protocol based on most commonly used interventions that led to improvements in balance. Our choice was specifically established on the assessment of the effects of balance training on postural control and injury prevention as well as balance training methods. The analyses including papers in which training protocols demonstrated positive effects on balance performance suggest that an efficient training protocol should last for 8 weeks, with a frequency of two training sessions per week, and a single training session of $45 \mathrm{~min}$. This standard was established based on 36 reviewed studies.

Key words: proprioceptive training, plyometrics, neuromuscular training, postural control, injury prevention.

\section{Introduction}

It has become almost routine practice to incorporate balance exercises into training programs for athletes from different sports, fall prevention programs for the elderly and rehabilitation programs. The objectives and benefits seem obvious, e.g., performance improvement and injury prevention as commonly cited goals (Hrysomallis 2011; Kümmel et al., 2016; Lesinski et al., 2015). However, the type of training that is most efficient still remains unclear, and the frequency, intensity and duration of exercise that would be most beneficial have not yet been determined. The main goal of this review was to establish whether a gold standard of balance training exists in this field.

Posture and balance control are fundamental in daily life to safely accomplish any type of movement and motor task that involves displacement of body segments or the entire body. Balance is the process of maintaining the body's center of gravity ( $\mathrm{CoG}$ ) vertically over the base of the support, and it relies on rapid and continuous feedback from visual, vestibular and somatosensory structures for the subsequent execution of smooth and coordinated neuromuscular actions (Winter, 1995; Zatsiorsky and Duarte, 1999). Efficient postural balance not only reduces the risk of body imbalance, fall, or subsequent injuries, but also contributes to the optimization of motor performance in a number of athletic disciplines (Hrysomallis, 2007; McGuine et al., 2000; Watson, 1999).

1 - Department of Human Motor Behavior, The Jerzy Kukuczka Academy of Physical Education, Katowice, Poland. 
Each sport involves specific motor skills that require the completion of particular postures and movements (Hrysomallis et al., 2006; Maurer et al., 2006; Paillard, 2017). Balance is an important factor in many athletic skills, but the relationship between sports competition results and balance is not yet fully understood (Adlerton et al., 2003; Hrysomallis, 2011). A lower level of balance is associated with injuries, such as sprains and, muscle, tendon and ligament strains among others (McGuine et al., 2000; Emery and Meeuwisse, 2010; Eils et al., 2010). Maintaining a standing posture on a stable surface is a major determinant of balance. A sway analysis in a simple task, such as quiet standing, is used as a variable of its description (Visser et al., 2008; Duarte and Freitas, 2010). However, controversy exists in the literature regarding the influence of balance training on athletes' performance and balance improvement, as well as injury prevention.

\section{Literature search}

The following review is based on papers that were found through computerized searches of PubMed and SportDiscus from 2000 to 2016. There is no general consensus in the literature regarding what to call training programs and exercise, therefore, we searched for various terms of training programmes. Based on a Boolean search strategy, consistent with previous meta-analyses on the effects of balance training (Kümmel et al., 2016; Lesinski et al., 2015; Zemková, 2014), the following search terms were (individually or in various combinations) used: "balance training" OR "proprioceptive training" OR "core stability training" OR "injury prevention", OR "postural control" AND "injury prevention" AND "sport" OR "athletes" OR "basketball" OR "baseball" OR "volleyball" OR "football" OR "soccer" OR "handball" OR "tennis" OR "ski" OR "runners" OR "judo" OR "taekwondo" OR "capoeira" OR "figure skating" OR "bicycling". The search was limited to English language and full-text original articles.

\section{Study selection}

Only the studies that met the following criteria were included: (1) the participants of an intervention and a control grouphad to be healthy at the time of the study, (2) the study subjects were in an age range of 7-30 years old, (3) balance tests were performed before and after the intervention programs. Studies were excluded if (1) they did not meet the criteria for CTs (Control Trials), (2) the PEDro scale was lower than four (Table 3), or (3) balance training was not described in detail. We made an exception for the four papers (PEDro 3) because of the better quality description of training protocols. The reviewers conducted the literature review independently, based on inclusion and exclusion criteria. In total, 50 studies met the inclusion criteria for review (Figure 1).

\section{Balance training}

\section{Training methods}

No general agreement may be found in the literature regarding which terms should be used to summarize training programs that aim at the improvement of postural stability (Kümmel, Kramer, Giboin, Gruber, et al., 2016). Some authors (Verhagen et al., 2005; Cumps et al., 2007; Kachanathu et al., 2014; Hammami et al., 2016) described balance or core stability exercises in their training programs. Others (Benis et al., 2016; Hammami et al.; Malliou et al., 2004; 2016; Pau et al., 2012; Verhagen et al., 2002; Zech et al., 2010) described neuromuscular or proprioceptive training and included multi-intervention programs with a combination of balance, strength, plyometric, and sport-specific exercises. Some authors describe the implemented exercises as balance training (Verhagen et al., 2005; Gioftsidou et al., 2006), and others call it sensorimotor training (Heleno et al., 2016; Pauet al., 2011), neuromuscular training (Zech et al., 2014; Benis et al., 2016) or proprioceptive training (Eils et al., 2010; Malliou et al., 2004; Mandelbaum et al., 2005). However, the most common term used seems to be balance training. Therefore, as in other systematic reviews (Kümmel et al., 2016), we use the term "balance training" to describe any training program primarily directed at the improvement of postural stability, regardless of the term used in the studies. Each of the training program described above presents a large variety of exercises. The balance training interventions consisted of balance exercises on both a stable and unstable surface, with or without recurrent destabilization during performance (Cumps et al., 2007; Hübscher et al., 2010; McHugh et al., 2007; Soderman et al., 2000; Verhagen et al., 2002, 2005; 
Zech et al., 2010). In some studies, training programs also included exercises with visual feedback (Malliou et al., 2004).

Frequently, studies that examined neuromuscular or proprioceptive training interventions similar to balance training included balance exercises on stable and unstable platforms with or without perturbations of postural control (Hübscher et al., 2010; Zech et al., 2010). Some authors also described neuromuscular training as multi-intervention programs with a combination of balance, weight, plyometric and sport-specific agility drills to address all aspects of neuromuscular control (Holm et al., 2004; Hübscher et al., 2010; Mandelbaum et al., 2005; Myer et al., 2009). In some papers, the authors implemented plyometric training alone to improve balance or combined it with balance exercises (Asadi et al., 2015; Manolopoulos et al., 2015; Myer et al., 2006; Pfile et al., 2013, 2016).

\section{Balance assessment}

To assess static and dynamic balance, some researchers used clinical and laboratory tests. Balance tests were performed before and after an intervention program. In some reports, also strength, aerobic endurance and specific performance were assessed (Hammami et al., 2016; Imai et al., 2014; Kang et al., 2013; Manolopoulos et al., 2015; Myer et al., 2006).

Static balance was evaluated using simple tests such us the stork test (Daneshjoo et al., 2012; Hammami et al., 2016) or the single leg stance (SLS) test (Dobrijević et al., 2016; Kang et al., 2013; Karami et al., 2014). These tests require the participants to keep their hands on the hips and maintain the foot of their non-tested leg at the knee level with their eyes open or closed. The participants attempted the task a few times, and the best scores were recorded for further analysis. More sophisticated procedures were performed on a force plate (FP) which can monitor the movement of the center of pressure (COP). Different variables derived from the path of the COP during the single leg stance test (Ahmadabadi et al., 2015; Malliou et al., 2004; Saunders et al., 2013), the quiet standing (QS) test (Cankaya et al., 2015; Pau et al., 2011; Steib et al., 2016) or the limit of stability (LOS) test (Mahieu et al., 2006; Romero-Franco et al., 2012) have been used as measures of balance. A balance assessment can also be conducted on an unstable surface. One example is a kinesthetic ability trainer (KAT) (Holm et al., 2004). The KAT consists of an electronic moveable platform that is supported by a small pivot at its central point. The stability of the platform is controlled by pressure that varies in a circular pneumatic bladder between the platform and the base of the unit. High pressure indicates an inflated platform (stable), while low pressure a deflated platform (unstable). An unstable surface makes the balance test more dynamic and possibly more applicable in a sports context.

Dynamic balance was assessed by the Balance Error Scoring System (BESS) (Imai et al., 2014; Mcleod et al., 2009), the Star Excursion Balance Test (SEBT) (Eisen et al., 2010; Filipa et al., 2012; Sato and Mokha, 2009) and the Y-Balance Test (YBT) (Trecroci et al., 2015; Benis et al., 2016; Hammami et al., 2016). The BESS consists of 6 separate $20 \mathrm{~s}$ balance tests that the subjects perform in different stances and on different surfaces. The test comprises 3 stance conditions (double-leg, single-leg, and tandem stance) and 2 surfaces (firm and foam). All trials are performed with the eyes closed (Finnoff et al., 2009). Errors are recorded as the quantitative measurement of postural stability under different testing conditions. Another test, originally described by Gray (Gray, 1995) as a rehabilitation tool, the SEBT, is a series of single-limb squats using the non-stance limb to perform maximal reach in order to touch a point along 1 of 8 designated lines on the ground. The lines are arranged in a grid that extends from a center point and are $45^{\circ}$ from one another. The reach distances are normalized to leg length. The YBT, was inspired by clinical applications of the SEBT (Coughlan et al., 2012). The participants push the reachindicator block with one foot in the anterior, posteromedial and posterolateral directions while standing on the other foot on a central footplate. Some researchers used the Modified Star Excursion Balance Test (MSEBT), where the subjects performed movements in the same directions as in the YBT (Zech et al., 2014; Heleno et al., 2016). Dynamic balance was also evaluated by a jumping test. For example, Heleno et al. (2016) conducted the Side Hop Test (SHT) with lateral jumps, and the Figure Eight Test (F8) using forward jumps with rotation. O'Malley et al. (2016) used the Landing Error Scoring System 
(LESS). The LESS identifies poor jump-landing techniques, such as decreased knee and hip flexion motion, knee valgus, and hip internal rotation, which can cause greater joint loading. Zech et al. (Zech et al., 2014) assessed the time to stabilization (TTS) following single-leg jump landing.

To assess proprioception and the stability of the upper and lower limbs, the isokinetic dynamometer (ID) was used to evaluate joint position sense (Daneshjoo et al., 2012).

\section{Equipment and exercises}

We found that there were numerous balance exercises that effectively improve static and dynamic balance. Training methods included exercises on stable and unstable surfaces in anterior/posterior and mediolateral directions, with or without recurrent destabilization (e.g., ball throwing or catching, strengthening exercises, or external perturbation applied by a partner) (Cumps et al., 2007; DiStefano et al., 2009; Hübscher et al., 2010; McHugh et al., 2007; Paillard, 2017; Soderman et al., 2000; Verhagen et al., 2002, 2005; Zech et al., 2010). The balance training programs typically included progression of the exercises. In some studies, balance exercises were performed first with the eyes open and then with the eyes closed in order to increase the difficulty (Hammami et al., 2016; Heleno et al., 2016; McGuine and Keene, 2006; Verhagen et al., 2005). Additionally, the balance training programs included transitions from a double-leg stance to a single-leg stance (Gioftsidou et al., 2006; O'Malley et al., 2016; Pau et al., 2011) on a stable or unstable surface (Eisen et al., 2010; Manolopoulos et al., 2015; Steib et al., 2016).

Occasionally, the authors also used exercises with visual feedback, such as moving a cursor to a target by shifting the weight (Malliou et al., 2004) or maintaining a single-leg stance on a board (Gioftsidou et al., 2006). For these types of exercises, the Biodex Stability System was used. In the studies, wobble boards that allow for multiplanar movement ( Eisen et al., 2010; Holm et al., 2004; Hrysomallis, 2007; Soderman et al., 2000), tilt boards permitting uniplanar movement (Dobrijević et al., 2016; Hrysomallis, 2007), BOSUs (Myer et al., 2006; Romero-Franco et al., 2012), foam mats (McHugh et al., 2007), inflated rubber discs (Saunders et al., 2013) and Swiss balls ( Kang et al., 2013; Sato and Mokha, 2009) were frequently used. These devices were used for different movements such as tilting, rotating, squatting, hopping, jumping, throwing and catching a ball (Eisen et al., 2010; Daneshjoo et al., 2012; Myer et al., 2006; Soligard et al., 2008). These activities were also combined with resistance exercises while balancing (Filipa et al., 2012; Petersen et al., 2005; Romero-Franco et al., 2012). In some papers, the authors implemented plyometric training alone to improve balance or combined plyometric training with balance exercises. These exercises emphasized jumping movements with feedback regarding technical performance and proper limb alignment (Asadi et al., 2015; Manolopoulos et al., 2015; Myer et al., 2006; Pfile et al., 2013, 2016). The plyometric exercises consisted of athletic positions, squat jumps, line jumps, bounding in place, and box drops, among others (Asadi et al., 2015; Myer et al., 2006). Core stability training was also used to improve balance. Some authors understood core exercises as bracing the abdominal muscles with low intensity limb movements (Kachanathu et al., 2014); however, most authors applied global training of larger superficial muscles around the abdominal and lumbar regions (Filipa et al., 2012; Iacono et al., 2014; Lust et al., 2009; Myer et al., 2006; Sato and Mokha, 2009). Core stability training included front planks, side planks, back bridges, quadruped exercises and exercises on a Swiss ball.

\section{The influence of balance training on balance in} various sport disciplines

The most widely studied disciplines were soccer (Cankaya et al., 2015; Daneshjoo et al., 2012; Gioftsidou et al., 2006; Imai et al., 2014), basketball (Asadi et al., 2015; Benis et al., 2016; Mcleod et al., 2009; Pfile et al., 2016) and handball (Holm et al., 2004; Karadenizli, 2016a, 2016b; Steib et al., 2016). The majority of the studies revealed significant differences between the groups after the interventions (Asadi et al., 2015; Daneshjoo et al., 2012; Kachanathu et al., 2014; Mcleod et al., 2009; O'Malley et al., 2016; Pfile et al., 2016; Steib et al., 2016). However, a few publications were found that did not show any significant influence of balance training on balance in various sport disciplines (Benis et al., 2016; Eisen et al., 2010; Sato and Mokha, 2009; Zech et al., 2014). 
Table 1

Influence of balance training on balance in various sports disciplines

\begin{tabular}{|c|c|c|c|c|c|c|c|c|c|c|}
\hline \multirow[b]{2}{*}{ References } & \multicolumn{3}{|c|}{ Subjects } & \multirow[b]{2}{*}{ Discipline } & \multicolumn{3}{|c|}{ Training Modality } & \multirow[b]{2}{*}{$\begin{array}{l}\text { Training } \\
\text { Type }\end{array}$} & \multirow[b]{2}{*}{$\begin{array}{c}\text { Device + } \\
\text { Procedure }\end{array}$} & \multirow[b]{2}{*}{ Conclusions } \\
\hline & N/Sex & Age (years) & $\begin{array}{c}\text { Status } \\
\text { Training }\end{array}$ & & $\begin{array}{c}\mathrm{D} \\
(\mathrm{min})\end{array}$ & $\begin{array}{c}F \\
\text { (n/week) }\end{array}$ & $\mathrm{T}$ (week) & & & \\
\hline Lust et al. (2009) & $\begin{array}{c}\text { IG: open } \\
\text { kinetic } \\
\text { chain/closed } \\
\text { kinetic chain } \\
\text { (OKC/CKC): } \\
\text { 12M, open } \\
\text { kinetic chain/ } \\
\text { closed kinetic } \\
\text { chain/core } \\
\text { stability } \\
\text { (OKC/CKC/CS } \\
\text { ): 13M } \\
\text { CG: } 15 \mathrm{M}\end{array}$ & $20.00 \pm 1.54$ & NR & baseball & $30-45$ & 3 & 6 & CST & $\begin{array}{c}\text { no device, } \\
\text { a single test } \\
\text { consisted of a } \\
\text { continuous } \\
\text { alternating } \\
\text { procedure to } \\
\text { lift one hand } \\
\text { to touch the } \\
\text { line then lift } \\
\text { the other } \\
\text { hand to touch } \\
\text { the line for } 15 \\
\mathrm{~s}\end{array}$ & $\begin{array}{l}\text { The OKC/CKC/CS } \\
\text { group and the } \\
\text { OKC/CKC group } \\
\text { demonstrated } \\
\text { significantly } \\
\text { greater scores than } \\
\text { the control group } \\
\text { after training. }\end{array}$ \\
\hline $\begin{array}{l}\text { Asadi et al. } \\
\text { (2015) }\end{array}$ & $\begin{array}{l}\text { IG (PLT): } 8 \mathrm{M} \\
\text { CG } \\
\text { (Basketball): } 8 \\
\text { M }\end{array}$ & $\begin{array}{c}\text { IG (PLT): } 20.1 \pm \\
0.8 \\
\text { CG : } 20.5 \pm 0.3\end{array}$ & amateur & basketball & 30 & 2 & 6 & PLT & SEBT & $\begin{array}{c}\text { After a 6-week } \\
\text { training period, } \\
\text { the PLT + BT } \\
\text { group showed } \\
\text { significant } \\
\text { improvements in } \\
\text { all directions, } \\
\text { whereas the } \\
\text { basketball group } \\
\text { did not show any } \\
\text { significant } \\
\text { changes. }\end{array}$ \\
\hline $\begin{array}{l}\text { Benis et al. } \\
\text { (2016) }\end{array}$ & $\begin{array}{l}\text { IG: } 14 \mathrm{~F} \\
\text { CG: } 14 \mathrm{~F}\end{array}$ & $\begin{array}{l}\text { IG: } 20 \pm 2 \\
\text { CG: } 20 \pm 1\end{array}$ & $\begin{array}{l}\text { national } \\
\text { league } \\
\text { players } \\
\text { practicing } 4 \\
\text { times a week } \\
\text { for } 2 \text { hours }\end{array}$ & basketball & 30 & 2 & 8 & NMT & YBT & $\begin{array}{l}\text { Improvement over } \\
\text { baseline scores } \\
\text { was noted in the } \\
\text { posteromedial and } \\
\text { posterolateral } \\
\text { reach directions } \\
\text { and in the } \\
\text { composite YBT } \\
\text { scores of the } \\
\text { experimental } \\
\text { group. No } \\
\text { differences in } \\
\text { anterior reach } \\
\text { were detected in } \\
\text { either group. } \\
\text { Differences were } \\
\text { noted in } \\
\text { postintervention } \\
\text { scores for } \\
\text { posteromedial } \\
\text { reach and } \\
\text { composite scores } \\
\text { between the } \\
\text { experimental and } \\
\text { control groups. }\end{array}$ \\
\hline $\begin{array}{l}\text { McLeod et al. } \\
\text { (2009) }\end{array}$ & $\begin{array}{l}\text { IG:37 F } \\
\text { CG: } 25 \mathrm{~F}\end{array}$ & $\begin{array}{l}\text { IG: } 15.6 \pm 1.1 \\
\text { CG: } 16.0 \pm 1.3\end{array}$ & competitive & basketball & 90 & 2 & 6 & $\begin{array}{c}\text { NMT } \\
\text { (functiona } \\
1 \\
\text { strengthen } \\
\text { ing, PLT, } \\
\text { agility BT) }\end{array}$ & $\begin{array}{l}\text { BESS } \\
\text { SEBT }\end{array}$ & $\begin{array}{l}\text { Trained subjects } \\
\text { scored } \\
\text { significantly fewer } \\
\text { BESS errors on the } \\
\text { single-foam and } \\
\text { tandem-foam } \\
\text { conditions at the } \\
\text { posttest than the } \\
\text { control group and } \\
\text { demonstrated } \\
\text { improvements on } \\
\text { the single-foam } \\
\text { compared with } \\
\text { their pretest, the } \\
\text { authors found a } \\
\text { significant } \\
\text { decrease in total } \\
\text { BESS errors in the } \\
\text { IG at the posttest } \\
\text { compared with } \\
\text { their pretest and } \\
\text { the CG. }\end{array}$ \\
\hline
\end{tabular}









\begin{tabular}{|c|c|c|c|c|c|c|c|c|c|c|}
\hline $\begin{array}{l}\text { Malliou et al. } \\
\text { (2004) }\end{array}$ & $\begin{array}{c}30 \text { (IG: } 15 \mathrm{M} / \mathrm{F} \\
\text { CG: } 15 \mathrm{M} / \mathrm{F})\end{array}$ & $19.3 \pm 9$ & $\begin{array}{c}\text { no } \\
\text { experience }\end{array}$ & skiing & 20 & 4 & NR & PT & $\begin{array}{l}\text { BBS: SLS } 20 \\
\text { s/3x (right, } \\
\text { left leg) }\end{array}$ & $\begin{array}{l}\text { No statistically } \\
\text { significant } \\
\text { differences } \\
\text { between the } \\
\text { groups were } \\
\text { found. }\end{array}$ \\
\hline $\begin{array}{l}\text { Karadenizli et } \\
\text { al. (2016) }\end{array}$ & $\begin{array}{l}\text { IG: } 14 \mathrm{~F} \\
\text { CG: } 12 \mathrm{~F}\end{array}$ & $\begin{array}{l}\text { IG: } 15.64 \pm 0.82 \\
\text { CG: } 15.38 \pm 0.92\end{array}$ & $\begin{array}{l}3.5 \text { years of } \\
\text { sport } \\
\text { experience }\end{array}$ & handball & $\mathrm{NR}$ & 2 & 10 & PLT & $\begin{array}{l}\text { FP: SLS (right, } \\
\text { left leg) }-30 \mathrm{~s}\end{array}$ & $\begin{array}{l}\text { The IG made } \\
\text { significantly } \\
\text { greater } \\
\text { improvements } \\
\text { than the CG in the } \\
\text { SLS (left). }\end{array}$ \\
\hline $\begin{array}{l}\text { Matin et al. } \\
\quad(2014)\end{array}$ & $\begin{array}{l}\text { IG: } 12 \mathrm{M} \\
\text { CG: } 12 \mathrm{M}\end{array}$ & $11.34 \pm 3.68$ & $\begin{array}{l}\text { the } \\
\text { representativ } \\
\text { e physical } \\
\text { fitness team } \\
\text { of } \\
\text { the } \\
\text { elementary } \\
\text { schools }\end{array}$ & fitness & 60 & 3 & 4 & NMT & $\begin{array}{c}\text { SLS 3x } \\
\text { Dynamic test: } \\
\text { (jumping) five } \\
\text { scores were } \\
\text { dedicated for } \\
\text { covering the } \\
\text { mark } \\
\text { and five } \\
\text { scores for } \\
\text { holding the } \\
\text { balance } \\
\text { stance as } \\
\text { static for } 5 \mathrm{~s}\end{array}$ & $\begin{array}{l}\text { Neuromuscular } \\
\text { training can } \\
\text { enhance important } \\
\text { factors of static } \\
\text { and dynamic } \\
\text { balance and the } \\
\text { results showed a } \\
\text { significant increase } \\
\text { in performance of } \\
\text { the individuals } \\
\text { participating in } \\
\text { neuromuscular } \\
\text { training. }\end{array}$ \\
\hline $\begin{array}{l}\text { Eisen et al. } \\
\text { (2010) }\end{array}$ & $\begin{array}{c}36 \mathrm{~F} / \mathrm{M} \\
\text { IG (dynadisc): } \\
12 \\
\text { IG (rocker } \\
\text { board): } 12 \text { CG: } \\
12\end{array}$ & $18-22$ & NR & $\begin{array}{c}\text { soccer/baske } \\
\text { tball }\end{array}$ & NR & 3 & 4 & BT & SEBT & $\begin{array}{c}\text { There was no } \\
\text { difference for each } \\
\text { group } \\
\text { individually, and } \\
\text { no difference } \\
\text { between trained } \\
\text { and untrained legs } \\
\text { within a subject }\end{array}$ \\
\hline $\begin{array}{l}\text { Zech et al. } \\
\text { (2014) }\end{array}$ & $\begin{array}{l}\text { IG: } 15 \\
\text { CG: } 15\end{array}$ & $\begin{array}{l}\text { IG: } 15.7 \pm 3.9 \\
\text { CG: } 14.1 \pm 1.4\end{array}$ & $\begin{array}{c}\text { first regional } \\
\text { youth } \\
\text { divisions }\end{array}$ & hockey & 20 & 2 & 10 & NMT & $\begin{array}{c}\text { FP: } 3 x \text { jump- } \\
\text { landing time } \\
\text { to } \\
\text { stabilization } \\
\text { (TTS), } \\
\text { SLS } 30 \mathrm{~s} / 3 \mathrm{x} \\
\text { (preferred } \\
\text { leg) } \\
\text { MSEBT } \\
\text { BESS }\end{array}$ & $\begin{array}{l}\text { All balance } \\
\text { measures except } \\
\text { the medial-lateral } \\
\text { TTS improved } \\
\text { significantly over } \\
\text { time in both } \\
\text { groups. Significant } \\
\text { group by time } \\
\text { interactions were } \\
\text { found for the BESS } \\
\text { score. The IG } \\
\text { showed greater } \\
\text { improvements } \\
\text { after } 10 \text { weeks of } \\
\text { training in } \\
\text { comparison to the } \\
\text { CG. }\end{array}$ \\
\hline $\begin{array}{l}\text { Myer et al. } \\
\text { (2006) }\end{array}$ & $\begin{array}{c}\text { IG (PLT): } 8 \mathrm{~F} \\
\text { IG2 (CST+BT): } \\
11 \mathrm{~F}\end{array}$ & $\begin{array}{l}\text { IG } 15.9+/-0.8 \\
\text { IG2 } 15.6+/-1.2\end{array}$ & $\begin{array}{c}\text { not less than } \\
4 \text { years of } \\
\text { experience }\end{array}$ & voleyball & 90 & 3 & 7 & $\begin{array}{l}\text { IG: PLT } \\
\text { IG2: } \\
\text { CST+BT }\end{array}$ & $\begin{array}{l}\text { FP: a single- } \\
\text { leg hop and } \\
\text { BT x3 } \\
\text { (randomized } \\
\text { trials on each } \\
\quad \text { side) }\end{array}$ & $\begin{array}{l}\text { The percentage } \\
\text { change from the } \\
\text { pretest to posttest } \\
\text { in vertical ground } \\
\text { reaction force was } \\
\text { significantly } \\
\text { different between } \\
\text { the PLT and } \\
\text { CST+BT groups } \\
\text { considering the } \\
\text { dominant side. }\end{array}$ \\
\hline $\begin{array}{l}\text { Romero-franco } \\
\text { et al. (2012) }\end{array}$ & $\begin{array}{l}\text { IG:12 F/M } \\
\text { CG: } 8 \text { F/M }\end{array}$ & $\begin{array}{c}\text { IG: } 37.75 \pm 10.63 \\
\text { CG: } 39.25 \pm \\
10.81\end{array}$ & $\begin{array}{l}\text { recreational } \\
\text { and } \\
\text { competitive }\end{array}$ & running & 30 & 3 & 6 & CST & $\begin{array}{c}\text { FP: QS (EO, } \\
\text { EC) } 2 \times 52 \mathrm{~s} \\
\text { BBS: EO } 3 \times 20 \\
\text { s, LOS in } 8 \\
\text { different } \\
\text { directions }\end{array}$ & $\begin{array}{l}\text { Significant } \\
\text { differences were } \\
\text { found in stability } \\
\text { in the medial- } \\
\text { lateral plane with } \\
\text { EO, gravity center } \\
\text { control in the right } \\
\text { direction and } \\
\text { gravity center } \\
\text { control in the back } \\
\text { direction after the } \\
\text { exercise } \\
\text { intervention in the } \\
\text { IG. } \\
\text { CST had no } \\
\text { significant } \\
\text { influence on scores } \\
\text { measured by the } \\
\text { SEBT or any GRF } \\
\text { variables. }\end{array}$ \\
\hline
\end{tabular}




\begin{tabular}{|c|c|c|c|c|c|c|c|c|c|c|}
\hline $\begin{array}{c}\text { Mahieu et al. } \\
\text { (2006) }\end{array}$ & $\begin{array}{l}\text { IG: } 6 \mathrm{~F}, 11 \mathrm{M} \\
\text { CG: } 8 \mathrm{~F}, 8 \mathrm{M}\end{array}$ & $9-15$ & competitive & skiing & 30 & 3 & 6 & VT & $\begin{array}{c}\text { SMART } \\
\text { Balance } \\
\text { Master: LOS } 8 \\
\text { s/8x, rhythmic } \\
\text { weight shift } \\
\text { left /right, } \\
\text { forward/back } \\
\text { ward }\end{array}$ & $\begin{array}{l}\text { No significant } \\
\text { differences except } \\
\text { for directional } \\
\text { control during the } \\
\text { LOS and the left- } \\
\text { right excursion of } \\
\text { the rhythmic } \\
\text { weight shift test } \\
\text { were found. }\end{array}$ \\
\hline $\begin{array}{c}\text { Cankaya et al. } \\
\text { (2015) }\end{array}$ & $\begin{array}{c}\text { IG: athletes } 25 \\
\text { M, sedentary: } \\
25 \mathrm{M} \\
\text { CG: } 25 \mathrm{M}\end{array}$ & 11 & NR & soccer & 40 & 3 & 8 & BT & $\begin{array}{c}\text { FP: QS }(E O, \\
\text { EC), } \\
\text { SLS }-30 \mathrm{~s} \\
\text { clockwise } \\
\text { rounds } 5 \times 60 \\
\text { s }\end{array}$ & $\begin{array}{c}\text { Balance } \\
\text { performance of the } \\
\text { athletes and } \\
\text { sedentary group } \\
\text { improved } \\
\text { compared to the } \\
\text { CG. }\end{array}$ \\
\hline $\begin{array}{l}\text { Daneshjoo et al. } \\
\text { (2012) }\end{array}$ & $\begin{array}{l}\text { CG: } 12 \mathrm{M} \text {; IG } \\
\text { (FIFA 11): } 12 \\
\text { M, IG } \\
\text { (HarmoKnee): } \\
12 \mathrm{M}\end{array}$ & $\begin{array}{c}\text { CG: } 19.7 \pm 1.6 \\
\text { IG FIFA 11: } \\
19.2 \pm 0.9 \\
\text { IG Ham o } \\
\text { Knee: } 17.7 \pm 0.4\end{array}$ & $\begin{array}{l}\text { professional } \\
\text { (five year } \\
\text { experience of } \\
\text { playing } \\
\text { soccer at } \\
\text { professional } \\
\text { level) }\end{array}$ & soccer & $20-25$ & 3 & 6 & $\begin{array}{c}\text { FIFA 11: } \\
\text { BT + ST + } \\
\text { PT } \\
\text { Harm o } \\
\text { Knee: BT + } \\
\text { ST + CST }\end{array}$ & $\begin{array}{c}\text { ID: JPS } \\
\text { SEBT } \\
\text { Stork Stand } \\
\text { Balance Test }\end{array}$ & $\begin{array}{l}\text { Both warm up } \\
\text { programs } \\
\text { improved } \\
\text { proprioception in } \\
\text { the dominant leg } \\
\text { at } 45^{\circ} \text { and } 60^{\circ} \text { knee } \\
\text { flexion. Dynamic } \\
\text { balance assessed } \\
\text { by the SEBT also } \\
\text { showed } \\
\text { improvement in } \\
\text { both groups, with } \\
\text { the HarmoKnee } \\
\text { group showing } \\
\text { significant } \\
\text { difference when } \\
\text { compared to the } \\
\text { CG. }\end{array}$ \\
\hline $\begin{array}{l}\text { Iacono et al. } \\
\quad(2014)\end{array}$ & $\begin{array}{l}\text { IG: } 10 \mathrm{M} \\
\text { CG: } 10 \mathrm{M}\end{array}$ & $\begin{array}{c}\text { IG: } 18.7 \pm 0.67 \\
\text { CG: } 19 \pm 0.63\end{array}$ & $\begin{array}{l}\text { competitive } \\
\text { players }\end{array}$ & soccer & NR & 4 & 5 & CST & $\begin{array}{l}\text { FP: SLS (EO, } \\
\text { EC) }-3 \times 20 \mathrm{~s} \\
\text { SEBT }\end{array}$ & $\begin{array}{l}\text { CST significantly } \\
\text { improved static } \\
\text { and dynamic } \\
\text { balance }\end{array}$ \\
\hline $\begin{array}{l}\text { Gioftsidou et al. } \\
\text { (2006) }\end{array}$ & $\begin{array}{l}39 \text { (CG:13, IG - } \\
\text { before } \\
\text { appropriate } \\
\text { training: } 13 \mathrm{M}, \\
\text { IG - after } \\
\text { appropriate } \\
\text { training: } 13 \text { ) }\end{array}$ & $16 \pm 1$ & $\begin{array}{l}\text { The young } \\
\text { championshi } \\
\text { p of the first } \\
\text { Greek } \\
\text { division }\end{array}$ & soccer & 20 & 3 & 12 & BT & $\begin{array}{l}\text { BBS: SLS } 20 \\
\text { s/3x (right, } \\
\text { left leg) }\end{array}$ & $\begin{array}{l}\text { Significant } \\
\text { differences in the } \\
\text { IG after training. }\end{array}$ \\
\hline $\begin{array}{l}\text { Heleno et al. } \\
\text { (2016) }\end{array}$ & $\begin{array}{l}\text { IG: } 12 \mathrm{M} \\
\text { CG: } 10 \mathrm{M}\end{array}$ & $14-16$ & $\begin{array}{l}\text { players with } \\
\text { a minimum } \\
\text { of } 3 \text { years of } \\
\text { training } \\
\text { experience; } \\
\text { participation } \\
\text { in state and } \\
\text { national } \\
\text { competitions; } \\
\text { training } 5 \\
\text { times a week }\end{array}$ & soccer & NR & 3 & 5 & SMT & $\begin{array}{c}\text { SLS, } \\
\text { Side Hop Test } \\
\text { (SHT), } \\
\text { Figure of } \\
\text { Eight Test } \\
\text { (F8) } \\
\text { MSEBT }\end{array}$ & $\begin{array}{l}\text { After a five-week } \\
\text { training program, } \\
\text { the intervention } \\
\text { group obtained } \\
\text { significant results } \\
\text { in the } \\
\text { F8, SHT and SEBT, } \\
\text { as well as in the } \\
\text { following } \\
\text { variables: area of } \\
\text { pressure of sway } \\
\text { center (COP), } \\
\text { mean velocity and } \\
\text { mean frequency of } \\
\text { COP }\end{array}$ \\
\hline $\begin{array}{c}\text { Trecroci et al. } \\
\text { (2015) }\end{array}$ & $\begin{array}{l}\text { IG: } 12 \mathrm{M} \\
\text { CG: } 12 \mathrm{M}\end{array}$ & $11.3 \pm 0.70$ & $\begin{array}{l}\text { sub-elite } \\
\text { players }\end{array}$ & soccer & 15 & 2 & 8 & BT & YBT & $\begin{array}{l}\text { Significantly } \\
\text { greater } \\
\text { improvements in } \\
\text { the YBT }\end{array}$ \\
\hline $\begin{array}{l}\text { Manolopoulos } \\
\text { et al. (2015) }\end{array}$ & $\begin{array}{c}\text { IG: } 20 \text { (ST: } 10 \\
\text { M } \\
\text { SMT: } 10 \mathrm{M} \text { ) }\end{array}$ & $\begin{array}{l}\text { ST: } 21.3 \pm 1.3 \\
\text { SMT: } 22 \pm 1.7\end{array}$ & amateur & soccer & NR & 2 & 8 & $\begin{array}{c}\text { ST } \\
\text { ST + SMT }\end{array}$ & $\begin{array}{c}\text { FP: stork } \\
\text { stance, raise } \\
\text { the heel off } \\
\text { the ground - } \\
5 \mathrm{~s}\end{array}$ & $\begin{array}{c}\mathrm{COP}(\mathrm{cm}) \text { in } \\
\text { anteriorposterior } \\
\text { and mediolateral } \\
\text { axes decreased } \\
\text { significantly after } \\
\text { training }\end{array}$ \\
\hline $\begin{array}{l}\text { Kachanathu et } \\
\text { al. (2014) }\end{array}$ & $\begin{array}{l}\text { IG: } 23 \mathrm{M} \\
\text { CG: } 23 \mathrm{M}\end{array}$ & $18 \pm 2$ & NR & soccer & 60 & $\begin{array}{c}\text { Phase-I: } \\
6 \\
\text { Phase-II: } \\
6 \\
\text { Phase- } \\
\text { III: } 3\end{array}$ & 4 & CST & $\begin{array}{c}\text { Double } \\
\text { Straight Limb } \\
\text { Lowering test: } \\
\text { x3 SEBT: } 8 \\
\text { directions x3 }\end{array}$ & $\begin{array}{c}\text { Significant } \\
\text { differences } \\
\text { of dynamic } \\
\text { balance and core } \\
\text { stability in the IG } \\
\text { compared to the } \\
\text { CG }\end{array}$ \\
\hline $\begin{array}{l}\text { Granacher et al. } \\
\text { (2016) }\end{array}$ & $\begin{array}{l}\text { IG: } 12 \mathrm{M} \\
\text { CG: } 12 \mathrm{M}\end{array}$ & $12-13$ & $\begin{array}{l}\text { first division } \\
\text { Tunisian }\end{array}$ & soccer & NR & 2 & 8 & $\begin{array}{l}\text { BT } \\
\text { PLT }\end{array}$ & $\begin{array}{c}\text { Standing } \\
\text { Stork Test, } \\
\text { YBT }\end{array}$ & $\begin{array}{l}\text { Results indicated } \\
\text { that BT provided } \\
\text { significantly } \\
\text { greater } \\
\text { improvements in } \\
\text { the YBT }\end{array}$ \\
\hline
\end{tabular}




\begin{tabular}{|c|c|c|c|c|c|c|c|c|c|c|}
\hline $\begin{array}{c}\text { Gioftsidou et al. } \\
\text { (2012) }\end{array}$ & $\begin{array}{l}\text { IG1: } 13 \\
\text { IG2: } 13 \\
\text { CG: } 12\end{array}$ & $22.7 \pm 3.5$ & $\begin{array}{l}\text { first Greek } \\
\text { division }\end{array}$ & soccer & 20 & $\begin{array}{l}\text { IG1: } 6 \\
\text { IG2: } 3\end{array}$ & $\begin{array}{l}\text { IG1: } 3 \\
\text { IG2: } 6\end{array}$ & BT & $\begin{array}{l}\text { BBS: SLS 20 s } \\
\text { x3 (each leg) } \\
\text { Balance } \\
\text { board: SLS } \\
\text { time to lose } \\
\text { balance }\end{array}$ & $\begin{array}{l}\text { Both training } \\
\text { groups } \\
\text { demonstrated } \\
\text { significant } \\
\text { improvements on } \\
\text { Biodex stability } \\
\text { tests. Similarly for } \\
\text { the balance board, } \\
\text { the results } \\
\text { revealed } \\
\text { significant } \\
\text { improvements for } \\
\text { both IGs. }\end{array}$ \\
\hline $\begin{array}{l}\text { Alyson et al. } \\
\text { (2012) }\end{array}$ & $\begin{array}{l}\text { IG: } 13 \mathrm{~F} \\
\text { CG: } 7 \mathrm{~F}\end{array}$ & $\begin{array}{l}\text { IG: } 15.4 \pm 1.5 \\
\text { CG:14.7 } \pm 0.8\end{array}$ & competitive & soccer & 50 & 2 & 8 & NMT & SEBT & $\begin{array}{l}\text { After NMT, } \\
\text { subjects } \\
\text { demonstrated a } \\
\text { significant } \\
\text { improvement in } \\
\text { the SEBT score on } \\
\text { the right and left } \\
\text { limb. }\end{array}$ \\
\hline $\begin{array}{c}\text { O'Malley et al. } \\
\text { (2016) }\end{array}$ & $\begin{array}{l}\text { IG: } 41 \mathrm{M} \\
\text { CG: } 37 \mathrm{M}\end{array}$ & $\begin{array}{c}\text { IG: } 18.6(18.4- \\
18.8) \\
\text { CG: } 18.3(18.1- \\
18.5)\end{array}$ & $\begin{array}{l}\text { teams were } \\
\text { required to } \\
\text { train at } \\
\text { least twice } \\
\text { per week. }\end{array}$ & $\begin{array}{l}2 \text { teams: } \\
1 \text { football } \\
1 \text { hurling }\end{array}$ & 15 & 2 & 8 & $\begin{array}{l}\text { GAA 15 } \\
\text { (Gaelic } \\
\text { Athletic } \\
\text { Associatio } \\
\text { n) training } \\
\text { program }\end{array}$ & $\begin{array}{c}\text { YBT } \\
\text { LESS } \\
\text { (Landing } \\
\text { Error Scoring } \\
\text { System) }\end{array}$ & $\begin{array}{l}\text { There was a } \\
\text { greater reduction } \\
\text { in mean LESS } \\
\text { score in favour of } \\
\text { the IG post } \\
\text { exercise training. } \\
\text { Clinically and } \\
\text { statistically } \\
\text { significant } \\
\text { improvements in } \\
\text { dynamic balance } \\
\text { and jump-landing } \\
\text { technique } \\
\text { occurred in } \\
\text { collegiate level } \\
\text { Gaelic football and } \\
\text { hurling players. }\end{array}$ \\
\hline Pau et al. (2012) & $\begin{array}{l}\text { IG: } 13 \mathrm{~F} \\
\text { CG: } 13 \mathrm{~F}\end{array}$ & $\begin{array}{l}\text { IG: } 13.2 \pm 0.2 \\
\text { CG: } 13.0 \pm 0.1\end{array}$ & $\begin{array}{l}0-3 \text { years of } \\
\text { experience }\end{array}$ & 更 & $20-30$ & $2-3$ & $6-9$ & NMT & $\begin{array}{c}\text { FP: QS } \\
(\mathrm{EO}, \mathrm{EC})-20 \mathrm{~s} \\
\text { SLS } 10 \mathrm{~s}\end{array}$ & $\begin{array}{l}\text { The IG exhibited } \\
\text { smaller sway areas } \\
\text { in EC conditions in } \\
\text { the bipedal stance, } \\
\text { while the other } \\
\text { variables were } \\
\text { unaffected. BT also } \\
\text { reduced sway area } \\
\text { and A-P COP } \\
\text { displacements of } \\
\text { the nondominant } \\
\text { limb for SLS. }\end{array}$ \\
\hline $\begin{array}{c}\text { Kang et al. } \\
\text { (2013) }\end{array}$ & $\begin{array}{c}36 \mathrm{M} \\
\text { IG (high school): } \\
8 \\
\text { IG (middle } \\
\text { school: } 8 \\
\text { CG (high } \\
\text { school): } 8 \\
\text { CG (middle } \\
\text { school): } 8\end{array}$ & NR & $\begin{array}{c}\text { middle } \\
\text { school: exp. } \\
\text { of } 25.44 \\
\text { months; high } \\
\text { school: exp. } \\
\text { of } 55.44 \\
\text { months }\end{array}$ & weightlifters & NR & NR & 8 & BT & SLS (EC) & $\begin{array}{l}\begin{array}{c}\text { Significant } \\
\text { changes }\end{array} \\
\text { were found in one- } \\
\text { leg standing time } \\
\text { with eyes closed in } \\
\text { the IG. }\end{array}$ \\
\hline
\end{tabular}

$N R=$ non reported; $I G=$ intervention group; $C G=$ control group; $\boldsymbol{F}=$ females; $\boldsymbol{M}=$ males $\boldsymbol{n}=$ group size; $\boldsymbol{P T}=$ prioproceptive training; $\boldsymbol{B} \boldsymbol{T}=$ balance training; $\boldsymbol{C S T}=$ core stability training; $\boldsymbol{P L T}=$ plyometric training; $S T=$ strength training; $S L S=$ single leg stance; $N M T=$ neuromuscular training; $D=$ training duration (min); $\boldsymbol{F}=$ frequency (n/week); $\boldsymbol{T}=$ duration of the intervention (week); $\boldsymbol{F} \boldsymbol{P}=$ force plate; $\boldsymbol{B B S}=$ biodex balance system; $\boldsymbol{S E B T}=$ star excursion balance test; $\boldsymbol{I D}=$ isokinetic dynamometry; $\boldsymbol{E} \boldsymbol{O}=$ eyes open; $E C=$ eyes close $\mathbf{Q S}=$ quiet standing; $B E S S=$ balance error scoring system; $Y B T=Y$ balance test $; S L L=$ single leg landing; $S M T=$ sensory motor training 
Table 2

Relationship between different balance prevention training and injuries

\begin{tabular}{|c|c|c|c|c|c|c|c|c|c|}
\hline \multirow[b]{2}{*}{ References } & \multicolumn{3}{|c|}{ Subjects } & \multirow[b]{2}{*}{ Discipline } & \multicolumn{3}{|c|}{ Training Modality } & \multirow[b]{2}{*}{$\begin{array}{l}\text { Training } \\
\text { Type }\end{array}$} & \multirow[b]{2}{*}{ Conclusions } \\
\hline & N/Sex & Age (years) & $\begin{array}{l}\text { Status } \\
\text { Training }\end{array}$ & & $\underset{(\min )}{\mathrm{D}}$ & $\begin{array}{c}\mathrm{F} \\
\text { (n/week) }\end{array}$ & $\mathrm{T}$ (week) & & \\
\hline Eils et al. (2010) & $\begin{array}{c}\mathrm{n}=198 \\
\mathrm{IG} 1=8149 \mathrm{M}: 32 \mathrm{~F} \\
\mathrm{CG} 1=9154 \mathrm{M}: 37 \mathrm{~F} \\
\mathrm{IG} 2=84 \mathrm{M}: 4 \mathrm{~F} \\
\mathrm{CG} 2=84 \mathrm{M}: 4 \mathrm{~F}\end{array}$ & $\begin{array}{c}\text { IG1 } \\
22.6 \pm 6.3 \\
\text { CG1 } \\
25.5 \pm 7.2 \\
\text { IG2 } \\
24.3 \pm 2.9 \\
\text { CG2 } \\
25.9 \pm 8.2\end{array}$ & $\begin{array}{l}\text { performance } \\
\text { level of the } \\
\text { players } \\
\text { varied } \\
\text { between the } \\
\text { seventh } \\
\text { highest } \\
\text { (Kreisliga) } \\
\text { and the } \\
\text { highest } \\
\text { league } \\
\text { (Bundesliga) } \\
\text { in Germany }\end{array}$ & basketball & 20 & 1 & $\begin{array}{c}\text { NR } \\
\text { (all } \\
\text { season) }\end{array}$ & PT & $\begin{array}{l}\text { The risk of sustaining an } \\
\text { ankle injury was } \\
\text { significantly reduced in } \\
\text { the IG by approximately } \\
35 \% \text {. The IG showed a } \\
\text { significantly more stable } \\
\text { SLS concerning the } \\
\text { mediolateral direction. } \\
\text { The degree of error for } \\
\text { 10- dorsiflexion and 15- } \\
\text { plantarflexion and the } \\
\text { mean error were } \\
\text { significantly reduced in } \\
\text { the posttest in the IG, but } \\
\text { not in the CG. }\end{array}$ \\
\hline $\begin{array}{l}\text { Soligard et al. } \\
\qquad(2008)\end{array}$ & $\begin{array}{l}\mathrm{n}=1892 \mathrm{~F} \\
\mathrm{IG}=1055 \\
\mathrm{CG}=837\end{array}$ & $13-17$ & $\begin{array}{l}\text { at least two } \\
\text { training } \\
\text { sessions a } \\
\text { week in } \\
\text { addition to } \\
\text { match play }\end{array}$ & soccer & 20 & NR & $\begin{array}{c}\text { NR } \\
\text { (8 months- } \\
\text { all season) }\end{array}$ & $\begin{array}{c}\text { Running } \\
\text { exercises } \\
\text { BT } \\
\text { CST } \\
\text { PLT }\end{array}$ & $\begin{array}{c}\text { There was a } \\
\text { significantly lower risk } \\
\text { of injuries overall, } \\
\text { overuse injuries, and } \\
\text { severe injuries in the IG. }\end{array}$ \\
\hline $\begin{array}{c}\text { Kraemer and } \\
\text { Knobloch (2009) }\end{array}$ & $\mathrm{IG}=24 \mathrm{~F}$ & $21 \pm 4$ & $\begin{array}{l}\text { German } \\
\text { premier } \\
\text { league }\end{array}$ & soccer & 3000 & NR & $\begin{array}{c}\mathrm{NR} \\
\text { (3 years) }\end{array}$ & $\begin{array}{l}\text { PT } \\
\text { BT } \\
\text { PLT }\end{array}$ & $\begin{array}{l}\text { One year after training } \\
\text { implementation, } \\
\text { noncontact injuries } \\
\text { decreased significantly } \\
\text { by } 65 \%(p=.021) . \\
\text { Overall, the mean injury } \\
\text { rate of all noncontact } \\
\text { injuries during all } \\
\text { intervention periods } \\
\text { significantly decreased } \\
\text { by } 42 \%(p=.045) \text { versus } \\
\text { the control period. }\end{array}$ \\
\hline $\begin{array}{l}\text { Owen et.al. } \\
\text { (2013) }\end{array}$ & $\begin{array}{c}\mathrm{n}=67 \mathrm{M} \\
\mathrm{IG}=44 \\
\mathrm{CG}=23\end{array}$ & $\begin{array}{l}\mathrm{IG}=28.6 \pm 3.75 \\
\mathrm{CG}=27.4 \pm 4.85\end{array}$ & $\begin{array}{l}\text { competitive } \\
\text { players }\end{array}$ & soccer & NR & 2 & $\begin{array}{c}\text { NR } \\
\text { (2 seasons: } \\
\text { 2008-2010) }\end{array}$ & $\begin{array}{l}\text { BT } \\
\text { ST } \\
\text { CST } \\
\text { FT }\end{array}$ & $\begin{array}{l}\text { During the intervention } \\
\text { season, the number of } \\
\text { muscle strain/tears was } \\
\text { less ( } 25 \% \text { of total } \\
\text { injuries) than the control } \\
\text { season ( } 52 \% \text { of total } \\
\text { injuries). }\end{array}$ \\
\hline $\begin{array}{c}\text { Timothy et al. } \\
\text { (2006) }\end{array}$ & $\begin{array}{c}\mathrm{n}=765 \mathrm{~F} / \mathrm{M} \\
\mathrm{IG}=373 \\
\mathrm{CG}=392\end{array}$ & $\begin{array}{l}\mathrm{IG}=16.4 \pm 1.2 \\
\mathrm{CG}=16.6 \pm 1.1\end{array}$ & $\begin{array}{l}\text { high school } \\
\text { students } \\
\text { trained by } \\
\text { certified } \\
\text { coaches }\end{array}$ & $\begin{array}{l}\text { basketball } \\
\text { soccer }\end{array}$ & 10 & 3 & $\begin{array}{c}\mathrm{NR} \\
\text { (all } \\
\text { season) }\end{array}$ & BT & $\begin{array}{l}\text { A reduced risk of an } \\
\text { ankle sprain was } \\
\text { observed after } \\
\text { intervention. }\end{array}$ \\
\hline Eils et al. (2010) & $\begin{array}{c}\mathrm{n}=198 \\
\mathrm{IG} 1=8149 \mathrm{M}: 32 \mathrm{~F} \\
\mathrm{CG} 1=9154 \mathrm{M}: 37 \mathrm{~F} \\
\mathrm{IG} 2=84 \mathrm{M}: 4 \mathrm{~F} \\
\mathrm{CG} 2=84 \mathrm{M}: 4 \mathrm{~F}\end{array}$ & $\begin{array}{c}\text { IG1 } \\
22.6 \pm 6.3 \\
\text { CG1 } \\
25.5 \pm 7.2 \\
\text { IG2 } \\
24.3 \pm 2.9 \\
\text { CG2 } \\
25.9 \pm 8.2\end{array}$ & $\begin{array}{l}\text { performance } \\
\text { level of the } \\
\text { players } \\
\text { varied } \\
\text { between the } \\
\text { seventh } \\
\text { highest } \\
\text { (Kreisliga) } \\
\text { and the } \\
\text { highest } \\
\text { league } \\
\text { (Bundesliga) } \\
\text { in Germany }\end{array}$ & basketball & 20 & 1 & $\begin{array}{c}\text { NR } \\
\text { (all } \\
\text { season) }\end{array}$ & PT & $\begin{array}{l}\text { The risk of sustaining an } \\
\text { ankle injury was } \\
\text { significantly reduced in } \\
\text { the IG by approximately } \\
35 \% \text {. The IG showed a } \\
\text { significantly more stable } \\
\text { SLS concerning the } \\
\text { mediolateral direction. } \\
\text { The degree of error for } \\
\text { 10- dorsiflexion and 15- } \\
\text { plantarflexion and the } \\
\text { mean error were } \\
\text { significantly reduced in } \\
\text { the posttest in the IG, but } \\
\text { not in the CG. }\end{array}$ \\
\hline $\begin{array}{l}\text { Soligard et al. } \\
\qquad(2008)\end{array}$ & $\begin{array}{l}\mathrm{n}=1892 \mathrm{~F} \\
\mathrm{IG}=1055 \\
\mathrm{CG}=837\end{array}$ & $13-17$ & $\begin{array}{l}\text { at least two } \\
\text { training } \\
\text { sessions a } \\
\text { week in } \\
\text { addition to } \\
\text { match play }\end{array}$ & soccer & 20 & NR & $\begin{array}{c}\text { NR } \\
\text { (8 months- } \\
\text { all season) }\end{array}$ & $\begin{array}{c}\text { Running } \\
\text { exercises } \\
\text { BT } \\
\text { CST } \\
\text { PLT }\end{array}$ & $\begin{array}{c}\text { There was a } \\
\text { significantly lower risk } \\
\text { of injuries overall, } \\
\text { overuse injuries, and } \\
\text { severe injuries in the IG. }\end{array}$ \\
\hline
\end{tabular}




\begin{tabular}{|c|c|c|c|c|c|c|c|c|c|}
\hline $\begin{array}{c}\text { Kraemer and } \\
\text { Knobloch (2009) }\end{array}$ & $\mathrm{IG}=24 \mathrm{~F}$ & $21 \pm 4$ & $\begin{array}{c}\text { German } \\
\text { premier } \\
\text { league }\end{array}$ & soccer & 3000 & NR & $\begin{array}{c}\text { NR } \\
\text { (3 years) }\end{array}$ & $\begin{array}{l}\text { PT } \\
\text { BT } \\
\text { PLT }\end{array}$ & $\begin{array}{l}\text { One year after training } \\
\text { implementation, } \\
\text { noncontact injuries } \\
\text { decreased significantly } \\
\text { by } 65 \%(p=.021) . \\
\text { Overall, the mean injury } \\
\text { rate of all noncontact } \\
\text { injuries during all } \\
\text { intervention periods } \\
\text { significantly decreased } \\
\text { by } 42 \%(p=.045) \text { versus } \\
\text { the control period. }\end{array}$ \\
\hline $\begin{array}{l}\text { Owen et.al. } \\
\text { (2013) }\end{array}$ & $\begin{array}{c}\mathrm{n}=67 \mathrm{M} \\
\mathrm{IG}=44 \\
\mathrm{CG}=23\end{array}$ & $\begin{array}{l}\mathrm{IG}=28.6 \pm 3.75 \\
\mathrm{CG}=27.4 \pm 4.85\end{array}$ & $\begin{array}{l}\text { competitive } \\
\text { players }\end{array}$ & soccer & NR & 2 & $\begin{array}{c}\text { NR } \\
\text { (2 seasons: } \\
\text { 2008-2010) }\end{array}$ & $\begin{array}{c}\text { BT } \\
\text { ST } \\
\text { CST } \\
\text { FT }\end{array}$ & $\begin{array}{l}\text { During the intervention } \\
\text { season, the number of } \\
\text { muscle strain/tears was } \\
\text { less ( } 25 \% \text { of total } \\
\text { injuries) than the control } \\
\text { season ( } 52 \% \text { of total } \\
\text { injuries). }\end{array}$ \\
\hline $\begin{array}{c}\text { Timothy et al. } \\
\text { (2006) }\end{array}$ & $\begin{array}{c}\mathrm{n}=765 \mathrm{~F} / \mathrm{M} \\
\mathrm{IG}=373 \\
\mathrm{CG}=392\end{array}$ & $\begin{array}{l}\mathrm{IG}=16.4 \pm 1.2 \\
\mathrm{CG}=16.6 \pm 1.1\end{array}$ & $\begin{array}{l}\text { high school } \\
\text { students } \\
\text { trained by } \\
\text { certified } \\
\text { coaches }\end{array}$ & $\begin{array}{l}\text { basketball } \\
\text { soccer }\end{array}$ & 10 & 3 & $\begin{array}{c}\text { NR } \\
\text { (all } \\
\text { season) }\end{array}$ & BT & $\begin{array}{l}\text { A reduced risk of an } \\
\text { ankle sprain was } \\
\text { observed after } \\
\text { intervention. }\end{array}$ \\
\hline $\begin{array}{l}\text { Malachy et al. } \\
\text { (2007) }\end{array}$ & $\begin{array}{c}\mathrm{n}=175 \\
\mathrm{IG}=175\end{array}$ & $15-18$ & $\begin{array}{l}\text { high school } \\
\text { students }\end{array}$ & football & 10 & 2 & 13 & $\begin{array}{c}\text { SLS } \\
\text { BT }\end{array}$ & $\begin{array}{l}\text { The injury incidence for } \\
\text { the players after the } \\
\text { intervention was } \\
\text { significantly lower than } \\
\text { the combined injury } \\
\text { incidence before the } \\
\text { intervention }(p<.01) \text {. }\end{array}$ \\
\hline $\begin{array}{l}\text { Cumps et } \\
\text { al.(2007) }\end{array}$ & $\begin{array}{c}\mathrm{n}=50 \mathrm{M} / \mathrm{F} \\
\mathrm{IG}=26 \\
\mathrm{CG}=24\end{array}$ & $\begin{array}{l}\mathrm{IG}=17.7 \pm 3.9 \\
\mathrm{CG}=18.0 \pm 2.7\end{array}$ & $\begin{array}{l}\text { elite youth } \\
\text { and young } \\
\text { senior } \\
\text { basketball } \\
\text { players }\end{array}$ & basketball & 10 & 3 & 22 & $\begin{array}{c}\text { BT } \\
\text { SLS } \\
\text { PLT } \\
\text { Dynamic } \\
\text { exercises }\end{array}$ & $\begin{array}{l}\text { Relative risks showed a } \\
\text { significantly lower } \\
\text { incidence of lateral ankle } \\
\text { sprains in the IG } \\
\text { compared to the CG. }\end{array}$ \\
\hline $\begin{array}{l}\text { Mandelbaum et } \\
\text { al. (2005) }\end{array}$ & $\begin{array}{l}\text { IG1: } 1041 \mathrm{~F} \\
\text { CG1: } 1905 \mathrm{~F} \\
\text { IG2: } 844 \mathrm{~F} \\
\text { CG2: } 1913 \mathrm{~F}\end{array}$ & $14-18$ & $\begin{array}{l}\text { competitive } \\
\text { female youth } \\
\text { soccer } \\
\text { players in a } \\
\text { southern } \\
\text { California } \\
\text { soccer league }\end{array}$ & soccer & 20 & NR & $\begin{array}{c}\text { NR } \\
\text { (2 season) }\end{array}$ & $\begin{array}{l}\text { Stretching } \\
\text { ST } \\
\text { PLT } \\
\text { Agility } \\
\text { NMT }\end{array}$ & $\begin{array}{l}\text { During the first period } \\
\text { (IG; CG1), there was an } \\
88 \% \text { decrease in ACL } \\
\text { injury in the IG subjects } \\
\text { compared to the control } \\
\text { group. In the second } \\
\text { period (IG2; CG2) there } \\
\text { was a } 74 \% \text { reduction in } \\
\text { ACL tears in the IG } \\
\text { compared to the age- } \\
\text { and skill-matched } \\
\text { controls. }\end{array}$ \\
\hline $\begin{array}{c}\text { Verhagen et al. } \\
\text { (2005) }\end{array}$ & $\begin{array}{c}\mathrm{n}=140 \mathrm{~F} \\
\mathrm{IG}=62 \\
\mathrm{CG}=78\end{array}$ & $\begin{array}{l}\mathrm{IG}=24.4 \pm 2.8 \\
\mathrm{CG}=24.2 \pm 2.5\end{array}$ & $\begin{array}{l}\text { the second } \\
\text { and third } \\
\text { Dutch } \\
\text { volleyball } \\
\text { divisions; } \\
\text { experience in } \\
\text { years } 13.3 \pm \\
2.3 \\
\text { players of the } \\
\text { second and } \\
\text { third } \\
\text { Swedish } \\
\text { divisions }\end{array}$ & voleyball & 15 & NR & $\begin{array}{c}\text { NR } \\
\text { (one } \\
\text { season } \\
\text { 2001/2002) }\end{array}$ & $\begin{array}{l}\text { BT } \\
\text { SLS }\end{array}$ & $\begin{array}{l}\text { Significantly fewer ankle } \\
\text { sprains in the IG were } \\
\text { found compared to the } \\
\text { CG. A significant } \\
\text { reduction in the ankle } \\
\text { sprain risk was found } \\
\text { only for players with a } \\
\text { history of ankle sprains. } \\
\text { The results showed no } \\
\text { significant differences } \\
\text { between the groups with } \\
\text { respect either to the } \\
\text { number, incidence, or } \\
\text { type of traumatic injuries } \\
\text { of the lower extremities. }\end{array}$ \\
\hline $\begin{array}{l}\text { Emery et al. } \\
\text { (2012) }\end{array}$ & $\begin{array}{c}\mathrm{n}=744 \mathrm{M} / \mathrm{F} \\
\mathrm{IG}=380 \\
\mathrm{CG}=364\end{array}$ & $\begin{array}{c}\text { IG: U13-15=46.6 } \\
\text { U16-18=53.4\% } \\
\text { CG: U13- } \\
\text { 15=48.9\% U16- } \\
18=51.1 \%\end{array}$ & $\begin{array}{l}\text { first and } \\
\text { second } \\
\text { Calgary } \\
\text { youth } \\
\text { division of } \\
\text { indoor } \\
\text { football }\end{array}$ & soccer & 30 & NR & 20 & $\begin{array}{c}\text { NMT } \\
\text { BT } \\
\text { ST } \\
\text { Agility } \\
\text { Stretching }\end{array}$ & $\begin{array}{l}\text { There was a } 38 \% \\
\text { reduction in all injury in } \\
\text { the IG compared with } \\
\text { the CG and a } 43 \% \\
\text { reduction in acute-onset } \\
\text { injury. }\end{array}$ \\
\hline $\begin{array}{l}\text { Hewett et al. } \\
\text { (1999) }\end{array}$ & $\begin{array}{c}\mathrm{n}=1263 \mathrm{~F} / \mathrm{M} \\
\mathrm{IG}=366 \mathrm{FCG}=463 \mathrm{~F} \\
\text { CGPopulation }=434 \\
\mathrm{M}\end{array}$ & $\begin{array}{l}\text { high school } \\
\text { students }\end{array}$ & $\begin{array}{l}\text { high school } \\
\text { students, } \\
\text { females were } \\
\text { players, } \\
\text { males were } \\
\text { not }\end{array}$ & $\begin{array}{c}\text { soccer, } \\
\text { basketball, } \\
\text { volleyball }\end{array}$ & $60-90$ & 3 & 6 & $\begin{array}{l}\text { NMT } \\
\text { PLT }\end{array}$ & $\begin{array}{l}\text { The untrained group } \\
\text { demonstrated an injury } \\
\text { rate } 3.6 \text { times higher } \\
\text { than the trained group } \\
\text { and } 4.8 \text { times higher } \\
\text { than the male control } \\
\text { group. The trained } \\
\text { group had a significantly } \\
\text { lower rate of noncontact } \\
\text { injuries than the } \\
\text { untrained group ( } p= \\
0.01) .\end{array}$ \\
\hline
\end{tabular}




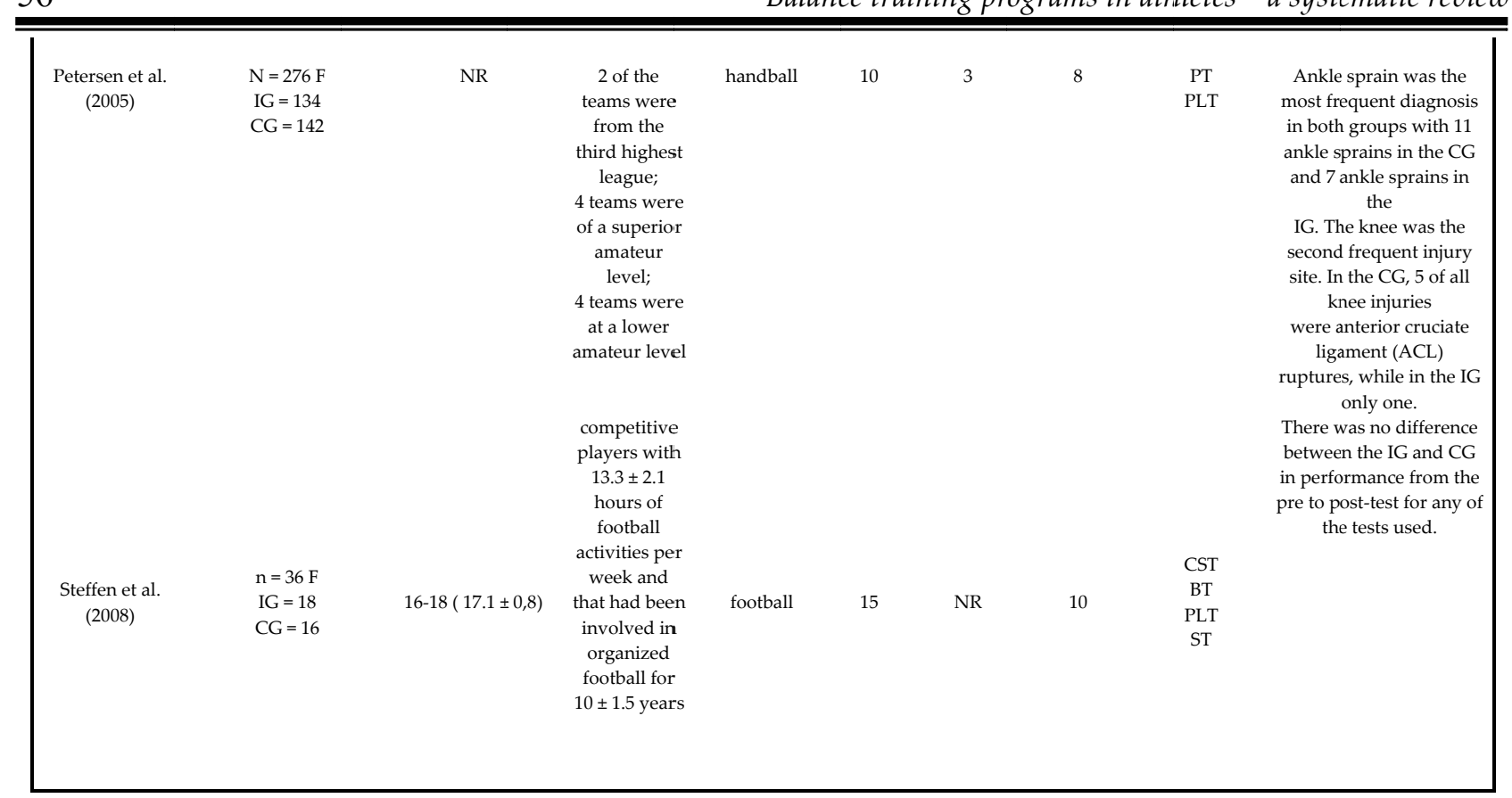

$N R=$ non reported; $I G=$ intervention group; $C G=$ control group; $\boldsymbol{F}=$ females; $\boldsymbol{M}=$ males; $\boldsymbol{n}=$ group size; $\boldsymbol{P T}=$ prioproceptive training; $\boldsymbol{B} \boldsymbol{T}=$ balance training; $\boldsymbol{C S T}=$ core stability training;

$P L T=$ plyometric training; $S T=$ strength training; $S L S=$ single leg stance; $N M T=$ neuromuscular training; $\boldsymbol{D}=$ training duration $(\mathrm{min}) ; \boldsymbol{F}=$ frequency $(\mathrm{n} /$ week); $\boldsymbol{T}=$ training duration (week)

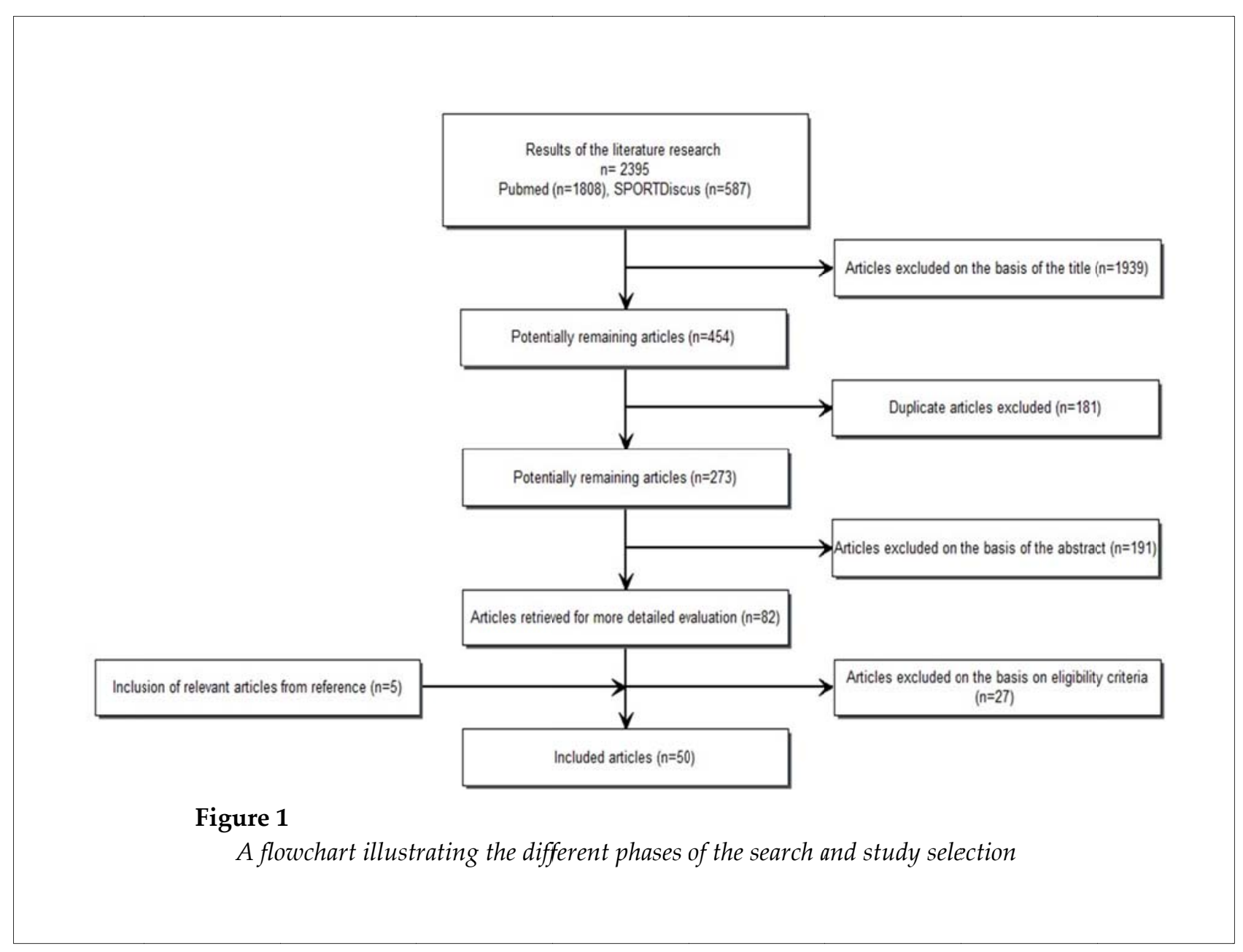


Table 3

Physiotherapy evidence database (PEDro) scores of the reviewed studies-

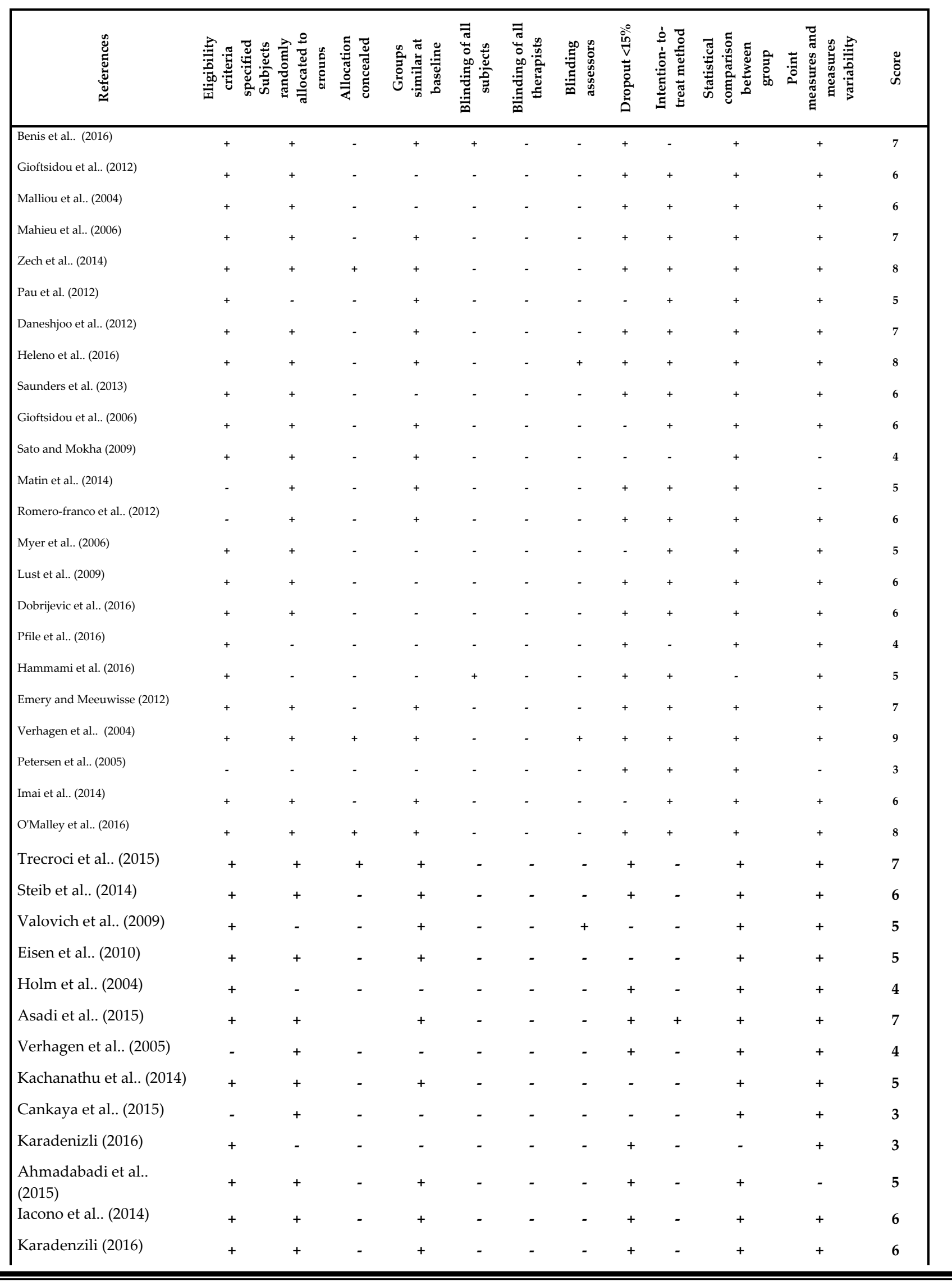




\begin{tabular}{|c|c|c|c|c|c|c|c|c|c|c|c|c|}
\hline Eils et al.. (2010) & + & + & - & + & - & - & - & + & + & + & + & 7 \\
\hline Soligard et al..(2008) & + & + & - & + & - & - & - & + & + & + & + & 7 \\
\hline $\begin{array}{l}\text { Kraemer and Knobloch } \\
(2009)\end{array}$ & - & - & - & - & - & - & - & + & - & + & + & 3 \\
\hline Owen et al.. (2013) & - & + & - & + & - & - & - & + & + & + & - & 5 \\
\hline $\begin{array}{l}\text { McGuine and Keene } \\
\text { (2006) }\end{array}$ & + & + & - & + & - & - & - & + & + & + & + & 7 \\
\hline McHugh et al.. (2007) & + & - & - & - & - & - & - & + & - & + & + & 4 \\
\hline Steffen et al.. (2008) & + & + & + & + & - & - & - & + & + & + & + & 8 \\
\hline Cumps et al.. (2007) & - & - & - & + & - & - & - & + & - & + & + & 4 \\
\hline $\begin{array}{l}\text { Mandelbaum et al.. } \\
(2005)\end{array}$ & - & - & - & - & - & - & - & + & + & + & + & 4 \\
\hline Soderman et al.. (2000) & + & + & - & + & - & - & - & - & - & + & + & 5 \\
\hline
\end{tabular}

\section{The influence of balance training on balance in various sport disciplines}

The most widely studied disciplines were soccer (Cankaya et al., 2015; Daneshjoo et al., 2012; Gioftsidou et al., 2006; Imai et al., 2014), basketball (Asadi et al., 2015; Benis et al., 2016; Mcleod et al., 2009; Pfile et al., 2016) and handball (Holm et al., 2004; Karadenizli, 2016a, 2016b; Steib et al., 2016). The majority of the studies revealed significant differences between the groups after the interventions (Asadi et al., 2015; Daneshjoo et al., 2012; Kachanathu et al., 2014; Mcleod et al., 2009; O'Malley et al., 2016; Pfile et al., 2016; Steib et al., 2016). However, a few publications were found that did not show any significant influence of balance training on balance in various sport disciplines (Benis et al., 2016; Eisen et al., 2010; Sato and Mokha, 2009; Zech et al., 2014).

The majority of the study interventions used full training units (Dobrijević et al., 2016; Filipa et al., 2012; Hewett et al., 1999; Kachanathu et al., 2014; Myer et al., 2006), but several authors applied balance training only as a warm-up (Ahmadabadi et al., 2015; Holm et al., 2004; O'Malley et al., 2016; Trecroci et al., 2015). Among the various exercise types, balance training and neuromuscular training (Eisen et al., 2010; Kang et al., 2013; Verhagen et al., 2005; Matin et al., 2014) were most commonly incorporated, followed by plyometric exercises and core stability training (Asadi et al., 2015; Karadenizli, 2016a, 2016b; Lust et al., 2009; Sato and Mokha, 2009).
To assess static balance, the authors mostly applied the SLS test $(\mathrm{n}=18)$ with 13 studies that used measurements conducted on a force plate. The most common procedure was the QS test $(n=16)$, and the LOS test was used twice. In the analyzed studies, the researchers mainly relied on the SEBT $(n=13)$, YBT $(n=4)$ and BESS $(n=2)$ to assess dynamic balance. Although the SEBT was the most popular in these studies, at least two difficulties accompanied this procedure. In many cases, the SEBT was assessed only in three directions that corresponded to the YBT (Filipa et al., 2012; Imai et al., 2014; Heleno et al., 2016). In addition, in several studies, the results were presented as composite reach scores (Daneshjoo et al., 2012; Eisen et al., 2010; Pfile et al., 2016). Therefore, even if the differences reached significance, it was not possible to ascertain the direction in which the improvement occurred. The detailed characteristics of the training protocols and tested tasks are shown in Table 1.

\section{The influence of balance training on injury prevention}

Balance control is a crucial factor in sports and an important component of common motor skills. Disturbances in balance control can increase the risk of injuries during high intensity activities (Burke-Doe et al., 2008; McGuine et al., 2000). The importance of balance control in the prevention of damage and musculoskeletal injuries during sports performance has been emphasized 
(Carolyn A Emery, 2005) and investigated in many studied cases (see review by Hrysomallis, 2007). Although the cause of injury is not always known, several risk factors for impairment in balance during training have been indicated (McKay et al., 2001). These factors include an insufficient warm-up (Woods et al., 2007), poor flexibility (Hartig and Henderson, 1999; Zakas et al., 2005), muscle imbalances (Parry and Drust, 2006; Croisier et al., 2008), muscle weakness (Croisier, 2004; Junge and Dvorak, 2004), neural tension (Turl and George, 1998), fatigue (Worrell, 1994), and previous injuries (Ekstrand et al., 2011; Parry and Drust, 2006).

The most common sports injuries $(60 \%)$ are sprains, dislocations, and ligament ruptures that occur at the knees and ankles and at the hands, elbows, and shoulders (Conn et al., 2003; Hawkins and Fuller, 1999; Powell and BarberFoss, 1999; Schneider et al., 2006). Improving balance in athletes by appropriate training has proven to engender positive effects on the reduction of the discussed injuries (Hrysomallis, 2007). Exercises may be included into a training program as part of an injury prevention strategy or with the primary goal of improving an athlete's performance (Sannicandro et al., 2014). According to Hrysomalis (2007), Hübscher (2010) and other authors, we are able to distinguish different design concepts and components of preventive exercises for balance including plyometrics, strengthening, balancing, endurance and stability, with a different approach to preventive programs (Heidt et al., 2000; Myklebust et al., 2003; Soderman et al., 2000). The results of our analysis of the relationship between different balance prevention training protocols and injuries are shown in Table 2. It indicates the effectiveness of balance training in reducing the incidence of sports injuries among athletes. The analysis of the prevention programs contains the results for team sports (such as basketball, soccer, handball, and volleyball), mainly because of their specificity (high-risk of injuries), which may consequently cause long-term disabilities for the injured player
(Lohmander et al., 2004; Myklebust and Bahr, 2005; von Porat et al., 2004).

\section{Conclusions}

In most of the reviewed articles, balance training has proven to be an effective tool for the improvement of postural control. However, a few articles stated that such effect did not occur (Eisen et al., 2010; Mahieu et al., 2006; Malliou et al., 2004; Sato and Mokha, 2009; Saunders et al., 2013; Verhagen et al., 2005), and a few studies, in which the effect was not reflected in all balance measures, suggested that balance training did not influence all of the dimensions of postural control (Benis et al., 2016; Holm et al., 2004; Pau et al., 2011; Zech et al., 2014). In some cases where the authors carried out both static and dynamic tests, significant results occurred in only one test type. Therefore, we would recommend the execution of both types of tests to decrease the risk of making inappropriate or global conclusions that training is ineffective in general.

Another issue is that the duration of training was heterogeneous. In most cases, it was approximately 40-50 min and was implemented as a full training session; however, in some articles, this time was rather short, i.e., only 10-20 min. In several studies, duration was not reported (Table 1). No gold standard is apparent in this field; therefore, it is difficult to make a global conclusion about the effectiveness of various types of balance training. Moreover, we are aware that it may be very difficult to establish one model of training that would be appropriate for each sport discipline, including its characteristics and demands. The main aim of this review was to identify a training protocol that is most commonly used and may lead to improvements in balance. Therefore, on the basis of analyses including papers in which training protocols resulted in positive effects on balance performance, it may be stated that an efficient training protocol should last for 8 weeks, with a frequency of two training sessions per week, and a single training session of $45 \mathrm{~min}$.

\section{Acknowledgements}

This research was supported by a grant of the Ministry of Science and Higher Education of Poland (RSA3 00953). 


\section{References}

Adlerton A-K, Moritz U, Moe-Nilssen R. Forceplate and accelerometer measures for evaluating the effect of muscle fatigue on postural control during one-legged stance. Physiother Res Int, 2003; 8(4): 187-199

Ahmadabadi F, Avandi S, Aminian-Far A. Acute versus chronic dynamic warm-up on balance and balance the vault performance in skilled gymnast. Int J Appl Exerc Physiol, 2015; 4(2): 20-33

Asadi A, Saez de Villarreal E, Arazi H. The effects of plyometric type neuromuscular training on postural control performance of male team basketball players. J Strength Cond Res, 2015; 29(7): 1870-1875

Benis R, Bonato M, Torre A La. Elite female basketball players' body-weight neuromuscular training and performance on the y-balance test. J Athl Train, 2016; 51(9): 688-695

Burke-Doe A, Hudson A, Werth H, Riordan DG. Knowledge of osteoporosis risk factors and prevalence of risk factors for osteoporosis, falls, and fracture in functionally independent older adults. J Geriatr Phys Ther, 2008; 31(1): 11-17

Cankaya S, Gokmen B, Tasmektepligil MY, Con M. Special balance developer training applications on young males- static and dynamic balance performance. Anthropologist, 2015; 19(1): 31-39

Conn JM, Annest JL, Gilchrist J. Sports and recreation related injury episodes in the US population, 1997-99. Inj Prev, 2003; 9(2): 117-123

Coughlan GF, Fullam K, Delahunt E, Gissane C, Caulfield BM. A comparison between performance on selected directions of the star excursion balance test and the $Y$ balance test., 2012; 47(4): 366-371

Croisier J-L. Factors associated with recurrent hamstring injuries. Sports Med, 2004; 34(10): 681-695

Croisier J-L, Ganteaume S, Binet J. Strength Imbalances and Prevention of Hamstring Injury in Professional Soccer Players: A Prospective Study. Am J Sports Med, 2008; 36: 1469-1475

Cumps E, Verhagen E, Meeusen R. Efficacy of a sports specific balance training programme on the incidence of ankle sprains in basketball. J Sport Sci Med, 2007; 6(2): 212-219

Daneshjoo A, Mokhtar AH, Rahnama N, Yusof A. The effects of comprehensive warm-up programs on proprioception, static and dynamic balance on male soccer players. PLoS One, 2012; 7(12): 1-10

DiStefano LJ, Clark MA, Padua DA. Evidence supporting balance training in healthy individuals: a systemic review. J Strength Cond Res, 2009; 23(9): 2718-2731

Dobrijević S, Moskovljević L, Dabović M. The influence of proprioceptive training on young rhythmic gymnasts balance. Facta Univ, 2016; 14(2): 247-255

Duarte M, Freitas SM. Revision of posturography based on force plate for balance evaluation. Rev Bras Fisioter, 2010; 14(3): 183-192

Eils E, Schröter R, Schröder M, Gerss J, Rosenbaum D. Multistation proprioceptive exercise program prevents ankle injuries in basketball. Med Sci Sports Exerc, 2010; 42(11): 2098-2105

Eisen T, Danoff J, Leone J, Miller T. The effects of multiaxial and uniaxial unstable surface balance training in college athletes. J Strength Cond Res, 2010; 24(7): 1740-1745

Ekstrand J, Hägglund M, Waldén M. Injury incidence and injury patterns in professional football - the UEFA injury study. Br J Sports Med, 2011; 45(7): 533-538

Emery CA. Injury prevention and future research. Individ Sport Med Sport Sci Basel, 2005; 48: 179-200

Emery CA, Meeuwisse WH. The effectiveness of a neuromuscular prevention strategy to reduce injuries in youth soccer: a cluster-randomised controlled trial. Br J Sports Med, 2010; 44(8): 555-562

Filipa A, Byrnes R, Paterno MV, Gregory D, Hewett TE. Neuromuscular training improves performance on the star excursion balance test in young female athletes. J Orthop Sport Phys Ther, 2012; 40(9): 551-558

Finnoff JT, Peterson VJ, Hollman JH, Smith J. Intrarater and interrater reliability of the balance error scoring system ( BESS ). PMER, 2009; 1(1): 50-54 
Gioftsidou A, Malliou P, Pafis G, Beneka A, Godolias G, Maganaris CN. The effects of soccer training and timing of balance training on balance ability. Eur J Appl Physiol, 2006; 96(6): 659-664

Gray GW. Lower extremity functional profile.Wynn, Marketing Incorporated; 1995

Hammami R, Granacher U, Makhlouf I, Behm DG, Chaouachi A. Sequencing effects of balance and plyometric training on physical performance in youth soccer athletes. J Strength Cond Res, 2016; 12: 3278-3289

Hartig DE, Henderson JM. Increasing hamstring flexibility decreases lower extremity overuse injuries in military basic trainees. Am J Sports Med, 1999; 27(2): 173-176

Hawkins R, Fuller C. A prospective epidemiological study of injuries in four English professional football clubs. Br J Sports Med, 1999; 33(3): 196-203

Heidt RS, Sweeterman LM, Carlonas RL, Traub JA, Tekulve FX. Avoidance of soccer injuries with preseason conditioning. Am J Sports Med, 2000; 28(5): 659-662

Heleno LR, da Silva RA, Shigaki L, Araujo CGA, Coelho Candido CR, Okazaki VHA, Frisseli A, Macedo C de SG. Five-week sensory motor training program improves functional performance and postural control in young male soccer players. A blind randomized clinical trial. Phys Ther Sport, 2016; 22: 74-80

Hewett TE, Lindenfeld T, Riccobene J, Noyes F. The effect of neuromuscular training on the incidence of knee injury in female athletes. A prospective study. Am J Sport Med Med, 1999; 27(6): 699-706

Holm I, Fosdahl MA, Friis A, Risberg MA, Myklebust G, Steen H. Effect of neuromuscular training on proprioception, balance, muscle strength, and lower limb function in female team handball players. Clin J Sport Med, 2004; 14(2): 88-94

Hrysomallis C. Relationship between balance ability, training and sports injury risk. Sport Med, 2007; 37(6): 547-556

Hrysomallis C. Balance ability and athletic performance. Sport Med, 2011; 41(3): 221-232

Hrysomallis C, McLaughlin P, Goodman C. Relationship between static and dynamic balance tests among elite Australian Footballers. J Sci Med Sport, 2006; 9(4): 288-291

Hübscher M, Zech A, Pfeifer K, Hänsel F, Vogt L, Banzer W. Neuromuscular training for sports injury prevention: a systematic review. Med Sci Sports Exerc, 2010; 42(3): 413-421

Iacono Dello A, Martone D, Alfieri A, Ayalon M. Core Stability Training Program (CSTP) effects on static and dynamic balance abilities. Gazz Med Ital, 2014; 173(4): 197-206

Imai A, Kaneoka K, Okubo Y, Shiraki H. Effects of two types of trunk exercises on balance and athletic performance in youth soccer players. Int J Sports Phys Ther, 2014; 9(1): 47-57

Junge A, Dvorak J. Soccer injuries: A review on incidence and prevention. Sport Med, 2004; 34(13): 929-938

Kachanathu S, Tyagi P, Anand P, Hameed U, Algarni A. Effect of core stabilization training on dynamic balance in professional soccer players. Phys Medizin, Rehabil Kurortmedizin, 2014; 24(6): 299-304

Kang SH, Kim CW, Kim Y IL, Kim KB, Lee SS, Shin KO. Alterations of muscular strength and left and right limb balance in weightlifters after an 8-week balance training program. J Phys Ther Sci, 2013; 25(7): 895-900

Karadenizli ZI. The effects of plyometric education trainings on balance and some psychomotor characteristics of school handball team. Univers J Educ Res, 2016; 4(10): 2286-2293

Karadenizli ZI. The effects of plyometric training on balance, anaerobic power and physical fitness parameters in handball. Anthropologist, 2016; 24(3): 751-761

Karami B, Ali M, Farzaneh Y, Homayoun G, Parmoon A. Neuromuscular training as the basis for developing the level of the static and dynamic balance in selected students of physical. Int J Sport Sci Fit, 2014; 4(1): 20-38

Kümmel J, Kramer A, Giboin L-S, Gruber M. Specificity of balance training in healthy individuals: a 
systematic review and meta-analysis. Sport Med, 2016; 46(9): 1261-1271

Lesinski M, Hortobagyi T, Muehlbauer T, Gollhofer A, Granacher U. Dose-response relationships of balance training in healthy young adults: a systematic review and meta-analysis. Sport Med, 2015; 45(4): 557576

Lohmander LS, Ostenberg A, Englund M, Roos H. High prevalence of knee osteoarthritis, pain, and functional limitations in female soccer players twelve years after anterior cruciate ligament injury. Arthritis Rheum, 2004; 50(10): 3145-3152

Lust KR, Sandrey MA, Bulger SM, Wilder N. The effects of 6-week training programs on throwing accuracy, proprioception, and core endurance in baseball. J Sport Rehabil, 2009; 18(3): 407-426

Mahieu NN, Witvrouw E, Van De Voorde D, Michilsens D, Arbyn V, Van Den Broecke W. Improving strength and postural control in young skiers: Whole-body vibration versus equivalent resistance training. J Athl Train, 2006; 41(3): 286-293

Malliou P, Amoutzas K, Theodosiou A, Gioftsidou A. Proprioceptive training for learning downhill skiing. Percept Mot Skills, 2004; 99(1): 149-154

Mandelbaum BR, Silvers HJ, Watanabe DS, Knarr JF, Thomas SD, Griffin LY, Kirkendall DT, Garrett WiJ. Effectiveness of a neuromuscular and proprioceptive training program in preventing the incidence of anterior cruciate ligament injuries in female athletes : 2-year follow up. Am J Sports Med, 2005; 33(7): 1003-1010

Manolopoulos K, Gissis I, Galazoulas C, Manolopoulos E, Patikas D, Gollhofer A, Kotzamandis C. Effect of combined sensorimotor-resistance training on strength, balance and jumping performance of soccer players. J Strength Cond Res, 2015; 30(1): 53-59

Matin KB, Yalfani A, Farzaneh G, Homayoun A, Parmoon A. Neuromuscular training as the basis for developing the level of the static and dynamic balance in selected students of physical. Int J Sport Sci Fit, 2014; 4(1): 20-38

Maurer C, Mergner T, Peterka RJ. Multisensory control of human upright stance. Exp Brain Res, 2006; 171(2): 231-250

McGuine TA, Greene JJ, Best T, Leverson G. Balance as a predictor of ankle injuries in high school basketball players. Clin J Sport Med, 2000; 10(4): 239-244

McGuine TA, Keene JS. The effect of a balance training program on the risk of ankle sprains in high school athletes. Am J Sports Med, 2006; 34(7): 1103-1111

McHugh MP, Tyler TF, Mirabella MR, Mullaney MJ, Nicholas SJ. The effectiveness of a balance training intervention in reducing the incidence of noncontact ankle sprains in high school football players. $\mathrm{Am}$ J Sports Med, 2007; 35(8): 1289-1294

McKay GD, Goldie PA, Payne WR, Oakes BW. Ankle injuries in basketball: injury rate and risk factors. Br J Sports Med, 2001; 35(2): 103-108

Mcleod TCV, Armstrong T, Miller M, Sauers JL. Balance improvements in female high school basketball players after a 6-week neuromuscular-training program. J Sport Rehabil, 2009; 18 465-481

Myer GD, Ford KR, McLean SG, Hewett TE. The effects of plyometric versus dynamic stabilization and balance training on lower extremity biomechanics. Am J Sports Med, 2006; 34(3): 445-455

Myer GD, Chu DA, Brent JE, Hewett TE. Of knee joint injury. Med Sci Sport, 2009; 27(3): 1-23

Myer GD, Ford KR, Brent JL, Hewett TE. The effects of plyometric versus dynamic stabilization and balance training on power, balance and landing force in female athletes. J Strength Cond Res, 2006; 20(2): 345353

Myklebust G, Bahr R. Return to play guidelines after anterior cruciate ligament surgery. BMJ, 2005; 39 127132

Myklebust G, Engebretsen L, Braekken IH, Skjølberg A, Olsen O-E, Bahr R. Prevention of anterior cruciate 
ligament injuries in female team handball players: a prospective intervention study over three seasons. Clin J Sport Med, 2003; 13(2): 71-78

O'Malley E, Murphy JC, McCarthy Persson U, Gissane C, Blake C. The effects of the GAA 15 training program on neuromuscular outcomes in Gaelic football and hurling players; a randomized cluster trial. J Strength Cond Res, 2016;

Paillard T. Plasticity of the postural function to sport and/or motor experience. Neurosci Biobehav Rev, 2017; 72 $129-152$

Parry L, Drust B. Is injury the major cause of elite soccer players being unavailable to train and play during the competitive season?. Phys Ther Sport, 2006; 7(2): 58-64

Pau M, Loi A, Pezzotta MC. Does sensorimotor training improve the static balance of young volleyball players?. Sport Biomech, 2011; 11(1): 97-107

Petersen W, Braun C, Bock W, Schmidt K, Weimann A, Drescher W, Eiling E, Stange R, Fuchs T, Hedderich J, Zantop T. A controlled prospective case control study of a prevention training program in female team handball players: the German experience. Arch Orthop Trauma Surg, 2005; 125(9): 614-621

Pfile KR, Gribble PA, Buskirk GE, Meserth SM, Pietrosimone BG. Sustained improvements in dynamic balance and landing mechanics after a 6-week neuromuscular training program in college women's basketball players. J Sport Rehabil, 2016; 25(3): 233-240

Pfile KR, Hart JM, Herman DC, Hertel J, Kerrigan DC, Ingersoll CD. Different exercise training interventions and drop-landing biomechanics in high school female athletes. J Athl Train, 2013; 48(4): 450-462

Powell JW, Barber-Foss KD. Injury patterns in selected high school sports: a review of the 1995-1997 seasons. J Athl Train, 1999; 34(3): 277-284

Romero-Franco N, Martinez-Lopez E, Lomas-Vega R, Hita-Contreras F, Martinez-Amat A. Effects of proprioceptive training program on core stability and center of gravity control in sprinters. J Strength Cond Res, 2012; 26(8): 2071-2077

Sannicandro I, Cofano G, Rosa RA, Piccinno A. Balance training exercises decrease lower-limb strength asymmetry in young tennis players. J Sport Sci Med, 2014; 13(2): 397-402

Sato K, Mokha M. Does core strength training influence running kinetics, lower-extremity stability and 5000m performance in runners? J Strength Cond Res, 2009; 23(1): 133-140

Saunders NW, Hanson NJ, Koutakis P, Chaudhari AM, Devor ST. Figure skater level moderates balance training. Int J Sports Med, 2013; 34(4): 345-349

Schneider S, Seither B, Tönges S, Schmitt H. Sports injuries: population based representative data on incidence, diagnosis, sequelae, and high risk groups. Br J Sports Med, 2006; 40(4): 334-339

Soderman K, Werner S, Pietilä T, Engström B, Werner S. Balance board training: prevention of traumatic injuries of the lower extremities in female soccer players? A prospective randomized intervention study. Knee Surgery, Sport Traumatol Arthrosc, 2000; 8: 356-363

Soligard T, Myklebust G, Steffen K, Holme I, Silvers H, Bizzini M, Junge A, Dvorak J, Bahr R, Andersen TE. Comprehensive warm-up programme to prevent injuries in young female footballers: cluster randomised controlled trial. BMJ, 2008; 337(2): 2469-2469

Steib S, Zahn P, zu Eulenburg C, Pfeifer K, Zech A. Time-dependent postural control adaptations following a neuromuscular warm-up in female handball players: a randomized controlled trial. BMC Sports Sci Med Rehabil, 2016; 8(1): 33-40

Trecroci A, Cavaggioni L, Caccia R, Alberti G. Jump rope training: Balance and motor coordination in preadolescent soccer players. J Sport Sci Med, 2015; 14(4): 792-798

Turl SE, George KP. A factor in repetitive hamstring strain?. J Orthop Sport Phys Ther, 1998; 27(1): 16-21

Verhagen E, Bobbert M, Inklaar M, Van Kalken M, Van Der Beek A, Bouter L, Van Mechelen W. The effect of a balance training programme on centre of pressure excursion in one-leg stance. Clin Biomech, 2005; 
20(10): 1094-1100

Verhagen E, van der Beek A, Twisk J, Bouter L, Bahr R, van Mechelen W. The effect of a proprioceptive balance board training program for the prevention of ankle sprains. Am J Sports Med, 2002; 32(6): 13851393

Visser JE, Carpenter MG, van der Kooij H, Bloem BR. The clinical utility of posturography. Clin Neurophysiol, 2008; 119(11): 2424-2436

von Porat A, Roos EM, Roos H. High prevalence of osteoarthritis 14 years after an anterior cruciate ligament tear in male soccer players: a study of radiographic and patient relevant outcomes. Ann Rheum Dis, 2004; 63(3): 269-273

Watson AWS. Ankle sprains in players of the field-games gaelic football and hurling. J Sport Med Phys Fit, 1999; 39(1): 66-70

Winter DA. Human balance and posture control during standing and walking. Gait Posture, 1995; 3(4): 193214

Woods K, Bishop P, Jones E. Warm-up and stretching in the prevention of muscular injury. Sport Med, 2007; 37(12): 1089-1099

Worrell T. Factors associated with hamstring injuries. An approach to treatment and preventive measures. Sport Med, 1994; 17(5): 338-345

Zakas A, Balaska P, Grammatikopoulou MG, Zakas N, Vergou A. Acute effects of stretching duration on the range of motion of elderly women. J Bodyw Mov Ther, 2005; 9(4): 270-276

Zatsiorsky VM, Duarte M. Instant equilibrium point and its migration in standing tasks: rambling and trembling components of the stabilogram. Motor Control, 1999; 3(1): 28-38

Zech A, Hübscher M, Vogt L, Banzer W, Hänsel F, Pfeifer K. Balance training for neuromuscular control and performance enhancement: a systematic review. J Athl Train Natl Athl Trainers' Assoc, 2010; 45(4): 392403

Zech A, Klahn P, Hoeft J, Zu Eulenburg C, Steib S. Time course and dimensions of postural control changes following neuromuscular training in youth field hockey athletes. Eur J Appl Physiol, 2014; 114(2): 395403

Zemková E. Sport-specific balance. Sport Med, 2014; 44(5): 579-590

\section{Corresponding author:}

\section{Kajetan J. Słomka}

Department of Human Motor Behavior

The Jerzy Kukuczka Academy of Physical Education in Katowice.

72A Mikolowska Street; 40-065 Katowice, Poland

tel +48 322075141

fax +48322516868

E-mail: k.slomka@awf.katowice.pl 